\title{
Generation of Rapid Sequences by Motor Cortex
}

Andrew J. Zimnik ${ }^{1,2}$ and Mark M. Churchland ${ }^{1,2,3,4 *}$

${ }^{1}$ Department of Neuroscience, Columbia University Medical Center, New York, New York, USA.

${ }^{2}$ Zuckerman Institute, Columbia University, New York, New York, USA.

${ }^{3}$ Kavli Institute for Brain Science, Columbia University Medical Center, New York, New York, USA.

${ }^{4}$ Grossman Center for the Statistics of Mind, Columbia University Medical Center, New York, New

*Correspondence: $\underline{\text { mc3502@columbia.edu }}$ 


\section{Abstract}

2 Rapid execution of motor sequences is believed to depend upon the fusing of movement elements into

3 cohesive units that are executed holistically. We sought to determine the contribution of motor cortex

4 activity to this ability. Two monkeys performed highly practiced two-reach sequences, interleaved with

5 matched reaches performed alone or separated by a delay. We partitioned neural population activity

6 into components pertaining to preparation, initiation, and execution. The hypothesis that movement

7 elements fuse makes specific predictions regarding all three forms of activity. We observed none of

8 these predicted effects. Instead, two-reach sequences involved the same set of neural events as

9 individual reaches, but with a remarkable temporal compression: preparation for the second reach

10 occurred as the first was in flight. Thus, at the level of motor cortex, skillfully executing a rapid sequence

11 depends not on fusing elements, but on the ability to perform two computations at the same time. 


\section{Introduction}

13 Decades of research have documented the events within motor cortex that accompany execution of a

14 voluntary movement. But what is 'a movement'? The answer is unambiguous for a single, discrete

15 behavior, such as pressing a button or reaching to a target. The subject begins a trial at rest, moves,

16 then returns to rest. 'A movement' is the action that occurs between periods of quiescence. But how

17 does this conception extend to other behaviors? For example, how many movements are used to enter

18 a four-digit bank code? If button presses are separated by considerable time, it seems reasonable to

19 assume they are generated individually. Conversely, when the sequence is well-learned and rapidly

20 executed, it seems likely to be generated as a unified whole.

21 There is indeed considerable evidence that the temporal separation between sequence elements relates

22 to how that sequence is internally produced ${ }^{1-3}$. When practiced in a specific order, the time between

23 elements becomes minimal, and they are said to form a functional unit: a 'motor chunk'1,3,4. Motor

24 chunk formation occurs spontaneously during long sequences, yielding subsets of swiftly executed

25 elements with longer separations between subsets ${ }^{1,5,6}$. A specific chunk structure can be encouraged by

26 manipulating the delay between elements ${ }^{7-9}$. Thus, chunking is both encouraged by, and a result of, the

27 need to rapidly execute multiple elements in sequence.

28 The neural and computational basis of this behavioral phenomenon is less clear. Is chunking a 'cognitive'

29 skill, relating to how groups of actions are recalled and conveyed to downstream motor areas? Or is

30 chunking a motor skill, in which motor areas generate, holistically, actions that were originally generated

31 separately? Studies of motor cortex report 'sequence selectivity': responses that reflect whether an

32 action is performed alone or within a sequence ${ }^{10-15}$ (although see ${ }^{16-18}$ ). Sequence selectivity concurs with

33 the hypothesis that chunking is a motor phenomenon, with chunks executed differently from their

34 component elements.

35 Yet sequence selectivity may arise for other reasons. Muscle activity can differ depending on whether an

36 action is performed within a sequence. This can be controlled for, but not perfectly. Sequence selectivity

37 could also result from overlap of execution-related and preparation-related activity ${ }^{19,20}$. If the next

38 element is prepared as the first is being completed, a 'new' pattern of activity, not observed during

39 execution of any single element, will be produced. For these reasons, one wishes to compare neural

40 activity not to a null hypothesis (identical responses regardless of whether an element occurs within a

41 sequence) but to predictions made by competing hypotheses. One hypothesis is that rapid production of 
42 a sequence does not depend on anything 'new' at the level of motor cortex, but simply reflects the

43 sequential preparation and execution of each element. The competing hypothesis is that elements are 44 prepared and executed as a unified whole.

45 The maturing characterization of neural events during single reaches makes it possible to derive 46 concrete predictions from these hypotheses. A reach involves three distinguishable neural processes

47 observable within motor and premotor cortex. The first is preparatory. Preparatory activity reflects the

48 identity of the pending movement and is proposed to seed subsequent movement-generating neural

49 dynamics ${ }^{21-26}$. The second is a putative 'trigger signal': a large change in neural state 150 ms before

50 movement onset ${ }^{27,28}$. This change does not reflect movement identity but rather the timing of

51 movement initiation. The third is execution-related: richly time-varying activity that arises $\sim 10-20 \mathrm{~ms}$

52 before muscle activity begins ${ }^{21,22}$. If two elements are prepared and executed sequentially, then

53 preparation-, triggering-, and execution-related events should occur twice. If two elements are prepared

54 and executed holistically, each event should occur once. These competing hypotheses also make

55 different predictions regarding the structure of neural activity during these stages. For example, before

56 the sequence begins, preparatory activity should reflect only the first element under the sequential

57 hypothesis, but should reflect aspects of both elements under the holistic hypothesis.

58 We recorded from premotor and primary motor cortex in monkeys trained to execute sequences of two

59 reaches, either in rapid succession (compound reach) or separated by an imposed pause (delayed

60 double-reach). As expected, the imposed pause produced clearly sequential preparation and execution:

61 the prepare-trigger-execute motif was observed twice. Unexpectedly, sequential preparation and

62 execution persisted during compound reaches. This was true even though the full sequence was known

63 in advance and had been practiced tens of thousands of times. It was true despite a very rapid pace, in

64 which muscle activity for the second reach began as the first was landing. Neural responses revealed

65 how this rapid pace was possible. Preparation for the second reach occurred during execution of the

66 first reach and did so without disrupting the first reach. Thus, at the level of motor and premotor cortex,

67 skilled performance depends not on fusing elements, but upon the ability to prepare one element while

68 executing another. 


\section{Results}

71 We trained two rhesus macaques (monkeys $\mathrm{B}$ and $\mathrm{H}$ ) to perform a modified delayed-reach task (Fig.

72 1a,b). All trials began with a randomized (0-1000 ms) instructed delay period. Each trial required the

73 monkey to make either a single reach, two reaches separated by an instructed pause (delayed double-

74 reach), or two reaches with no pause between them (compound reach). Target color indicated which

75 should be acquired first. A salient visual cue (the diameter of the colored portion of the first target)

76 indicated whether a pause was required. For monkey $\mathrm{H}$, the pause between delayed double-reaches was

77 always $600 \mathrm{~ms}$. For monkey B it was variable (100, 300, or $600 \mathrm{~ms}$; the last is used for most analyses) and

78 indicated by the visual cue. Thus, all key information - target locations and any instructed pause - was

79 given during the instructed delay. Reach paths are illustrated (Fig. 1a) for all single reaches, for three

80 compound reaches that began down-and-right, and for three compound reaches that began down-and-

81 left (Extended Data Fig. 1 shows paths for all compound reaches).

82 Compound reaches were performed briskly; the hand stayed on the first target only briefly before

83 moving to the second target. Median dwell times on the first target were $119 \mathrm{~ms}$ (monkey B) and 137

$84 \mathrm{~ms}$ (monkey H). The median duration for the full two-reach sequence was $561 \mathrm{~ms}$ (monkey B) and 645

85 ms (monkey $\mathrm{H}$ ). This rapid pace resulted from extensive training over months, with each sequence

86 performed tens of thousands of times. This pace is even faster than that reported in other motor

87 sequence tasks performed by non-human primates, where dwell times are typically $>200 \mathrm{~ms}^{10,11,29}$.

To enable comparisons at the neural level, every compound reach condition shared the same first target

89 with a matched single-reach condition. Comparisons at the neural level are most interpretable if the

90 reaches themselves (not just target locations) are well-matched. This was indeed the case. Reach-speed

91 profiles (Fig. 1c,d) were very similar for single reaches (black) and the first reach of compound reaches

92 (gray); correlations were $0.99 \pm 0.002$ (mean \pm standard deviation, monkey B) and $0.98 \pm 0.03$ (monkey

$93 \mathrm{H})$. The most noticeable difference was that the first reach of a compound reach tended to be slightly

94 faster than the corresponding single reach: by $3 \pm 2.8 \%$ and $3 \pm 9.4 \%$ (monkey $B$ and $H$, change in peak

95 velocity averaged across conditions). We also examined the activity of the major muscles of the upper

96 arm and shoulder (Fig. 2a and Extended Data 3 show two such recordings). Muscle activity began

97 changing $100 \mathrm{~ms}$ before reach onset (circles). During the subsequent $275 \mathrm{~ms}$, muscle activity during 
bioRxiv preprint doi: https://doi.org/10.1101/2020.06.09.143040; this version posted June 10, 2020. The copyright holder for this preprint (which was not certified by peer review) is the author/funder, who has granted bioRxiv a license to display the preprint in perpetuity. It is made available under aCC-BY-NC-ND 4.0 International license.

\section{Fig. 1}

a
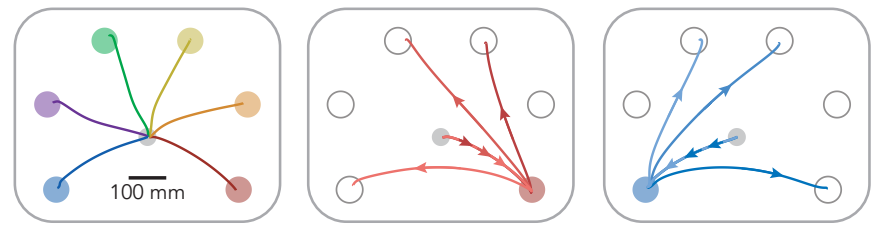

b
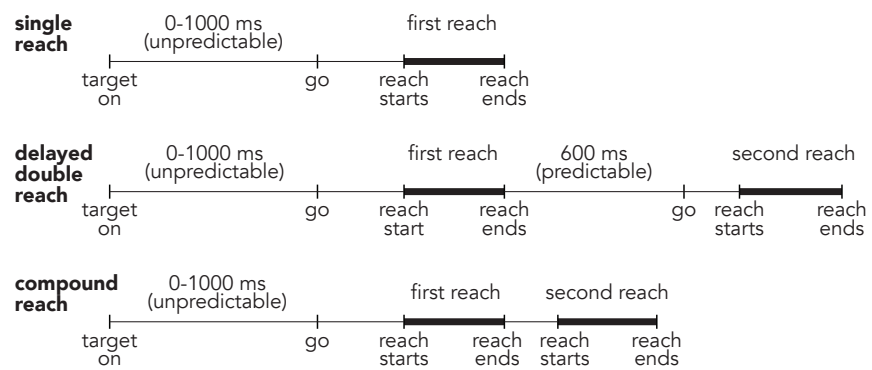

c

d
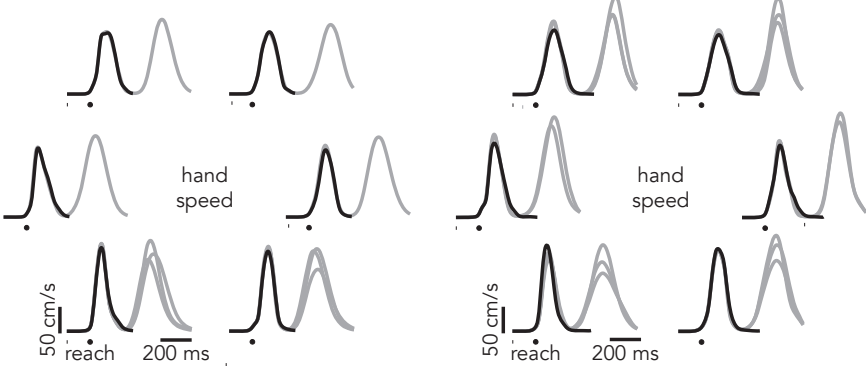

Fig. 1 | Task structure. a, Mean hand paths for all single reach conditions and a subset of compound reach conditions. Left panel: single reaches. Middle panel: three compound reaches that began with a reach to the bottom-right target. Right panel: three compound reaches that began with a reach to the bottom-left target. $\mathbf{b}$, Trial timing. Single reaches employed a variable instructed delay period. Delayed double-reaches employed an instructed pause between reaches, the duration of which was indicated during the initial instructed delay. Compound reaches had no instructed pause and monkeys reached for the second target immediately after the first was touched. c, Mean hand speed for all single reach (black) and compound reach (grey) conditions. Traces are grouped according to the location of the first target. Every target location served as the first target for at least one compound reach condition; some were used for three. Averages were calculated across all trials and recording sessions. Data are for monkey B. d, Same as $\mathbf{c}$ but for Monkey H. Every target location served as the first target for two or three compound reach conditions. 
compound reaches was similar to that during single reaches to the same target (gray and black traces

99 overlap). Mean correlations were $0.93 \pm 0.15$ and $0.93 \pm 0.15$ (monkey B and $\mathrm{H}$, average across muscles and conditions). Comparing compound reaches with matched single reaches, muscle activity magnitude was, on average, slightly lower for monkey B ( $2 \pm 15 \%)$ and slightly higher for monkey H $(8 \pm 22 \%)$. Thus,

102 behavior approached the desired ideal: an identical first reach regardless of a second reach. The match was even closer when comparing delayed double-reaches with single reaches, both for velocity $(\rho=0.99$

$104 \pm 0.0004$ and $0.99 \pm 0.001$ monkey $B$ and $H$ ) and muscle activity (average $\rho=0.98 \pm 0.05$ and $0.99 \pm$ 105 0.05).

The final relevant comparison is between compound and delayed double-reaches, which would ideally

107 have identical second reaches. This is probably impossible; during compound reaches the second reach

108 is generated while muscle activity is still in flux after the first reach (e.g., post-reach co-contraction).

109 Consistent with this, second-reach muscle activity was strongly but imperfectly correlated between compound and delayed double-reaches: $\rho=0.90 \pm 0.16$ and $0.83 \pm 0.25$ (monkey $B$ and $H$, average

111 across muscles and conditions).

112 During compound reaches, generation of the second reach requires muscle activity, at some point, to 113 depart strongly from that during single reaches. This occurred $\sim 275 \mathrm{~ms}$ after initial EMG onset. This

114 translates to $\sim 25 \mathrm{~ms}$ before the end of the first reach and $150 \mathrm{~ms}$ before the onset of the second reach.

115 This timing underscores how rapidly compound reaches unfolded - muscle activity began changing in

116 anticipation of the second reach as the first reach was ending. As a result, muscle activity during

117 compound reaches evolved continuously throughout the full sequence; there was no pause or plateau

118 between reaches.

\section{Basic Properties of Neural Responses}

120 We recorded activity from the arm region of motor cortex (dorsal premotor cortex and surface primary 121 motor cortex, Extended Data Fig. 2) using 32-channel linear-array electrodes. Recordings consisted of 122 well-isolated single neurons and high-quality multi-unit isolations (227 and 587 units from monkey B and $123 \mathrm{H})$. As in prior studies ${ }^{10-13,30}$ many units exhibited sequence selectivity - i.e., responses associated with a 124 reach could depend on whether it was part of a sequence. Comparing delay-period activity before 125 matched single versus compound reaches, $11 \%$ and $24 \%$ of recorded units (monkey B and H) showed 126 significantly different responses during at least one pair of conditions $(p<0.001$, unpaired t-test, 
127 adjusted for multiple comparisons). These percentages grew when comparing movement-epoch activity:

128 to $38 \%$ and $60 \%$ (when considering time-averaged activity) or $79 \%$ and $95 \%$ (when considering the full 129 temporal pattern; see Methods).

130 Such differences are potentially consistent with a holistic strategy, which requires that an individual 131 reach be generated 'differently' when part of a sequence. Had we found no significant differences, a 132 holistic strategy could therefore have been rejected. Yet the mere presence of significant differences 133 does not discriminate between sequential and holistic strategies. Muscle activity also differs slightly, 134 rendering small but potentially significant neural differences unavoidable. Furthermore, sequence 135 selectivity is potentially expected during movement even under the sequential hypothesis. If preparation for the second reach overlaps with execution of the first, that would create a 'new' pattern

137 of activity not observed during any single reach. Thus, distinguishing between holistic and sequential 138 strategies requires testing more specific predictions.

140 Such predictions are possible because of extensive prior characterization of neural activity in motor 141 cortex during single reaches ${ }^{19,20,31-34}$. This has yielded a paradigm in which reach generation is proposed 142 to be subserved by three internal processes: preparation, triggering, and execution ${ }^{21-26,35-37}$. Preparatory 143 activity, typically observed during an instructed delay, has long been hypothesized to be a necessary 144 precursor of voluntary movement ${ }^{19,20,31}$. Importantly, preparatory activity also occurs without an 145 instructed delay ${ }^{21,31,35,38}$ and can develop very rapidly ${ }^{21,35}$. Preparatory activity is proposed to seed 146 execution-related activity and thus specify the identity of the executed reach. The transition from 147 preparatory to execution-related activity coincides with a large condition-invariant change in the neural 148 state, proposed to result from a movement-triggering input ${ }^{27,39-41}$. Traditionally it has been challenging 149 to discriminate preparation-related activity from execution-related activity when they occur close in 150 time ${ }^{31}$. However, the recent focus of neural subspaces ${ }^{42-44}$ - sets of neural dimensions that capture 151 prominent response features - opens up new approaches. In particular, preparation-related, triggering152 related, and execution-related activity occur in nearly orthogonal subspaces ${ }^{21,27,28,36}$ and can thus be 153 separated via linear projections of the population response.

154 The above paradigm makes it possible to concretely specify the competing hypotheses and derive their 155 predictions. Under the holistic strategy (Fig. 2b) the two elements of a compound reach are produced 
bioRxiv preprint doi: https://doi.org/10.1101/2020.06.09.143040; this version posted June 10, 2020. The copyright holder for this preprint (which was not certified by peer review) is the author/funder, who has granted bioRxiv a license to display the preprint in perpetuity. It is made available under aCC-BY-NC-ND 4.0 International license.

\section{Fig. 2}

a
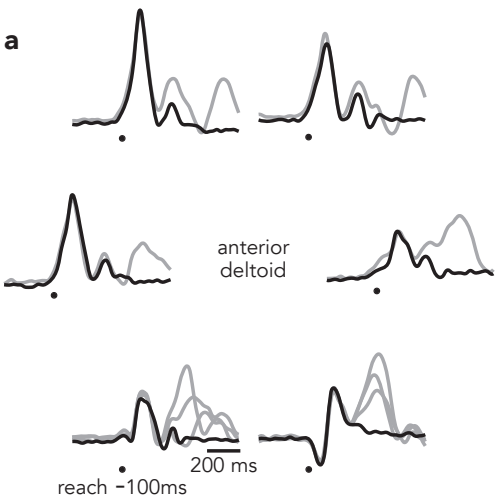

b
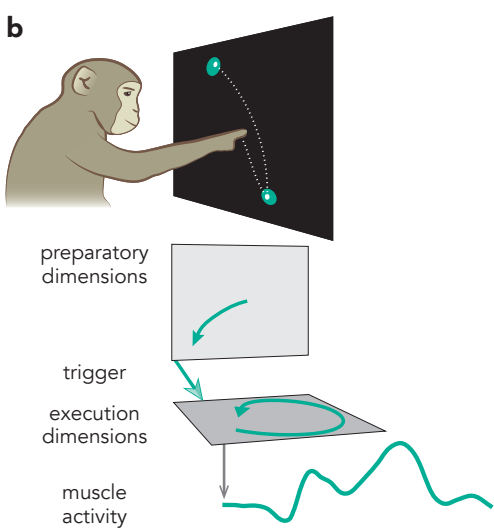

c

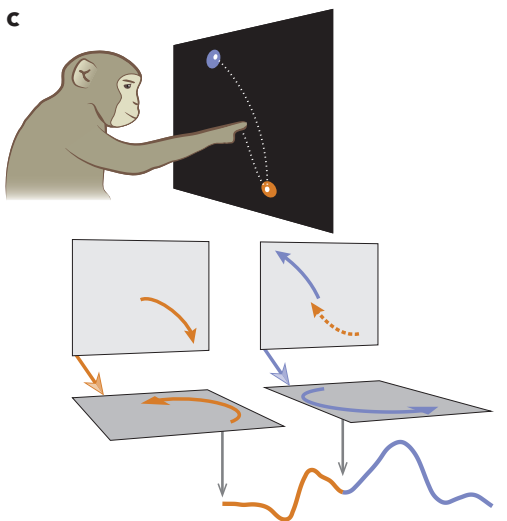

Fig. 2 | Potential strategies for generating compound reaches. a, Mean EMG for single (black) and compound (grey) reach conditions. Data are from the deltoid of Monkey B. Traces are grouped according to the location of the first target. $\mathbf{b}$, Illustration of the holistic strategy. Under the holistic strategy, compound reaches are produced as single movements, which require only a single pattern of preparatory activity and a single trigger signal. c, Illustration of the sequential strategy. Under the sequential strategy, compound reaches are generated by preparation, triggering, and execution occurring twice in succession. 
cohesively, as if they were a single movement. An appropriate preparatory state is established, a trigger

157 signal arrives, and execution-related dynamics generate activity that produces the desired continuous

158 pattern of muscle activity. Under the sequential strategy (Fig. 2c), a compound reach is simply two distinct movements, prepared and executed sequentially. The first is prepared, triggered, and executed as if it were a single reach. Shortly thereafter, these three stages occur a second time. Continuous

161 muscle activity results from the concatenated output.

162 The holistic and sequential hypotheses yield three mutually exclusive predictions. First, preparatory 163 activity should occur once if the holistic strategy is employed and twice if the sequential strategy is 164 employed. Second, under the holistic strategy, preparatory activity before a compound reach should differ from that before a corresponding single reach. In contrast, the sequential strategy predicts that preparatory activity will be similar, because only the first reach is prepared at that time. Third, the

167 holistic strategy predicts a single trigger-related change in activity, whereas the sequential strategy 168 predicts that each reach will be preceded by its own trigger-related event.

169 While it is hoped that observations will consistently obey all the predictions of one hypothesis, it is also possible for results to be mixed or fail to follow any of the above predictions. Thus, evaluating these

171 predictions provides not only a test of the two hypotheses, but of the paradigm itself. Partly for this

172 reason, our task also included delayed double-reaches as a reference. The imposed pause between the

173 two reaches, instructed during the initial delay, is expected to dissuade the use of a holistic strategy.

174 Delayed double-reaches thus afford an opportunity to confirm the predictions of the sequential strategy 175 in a context where it is likely to be used. This provides a foundation for asking what occurs when there is 176 no pause and the holistic strategy becomes viable.

\section{Timing of Single-Neuron Preparatory Activity}

178 Most of the above predictions must be tested at the population level because most neurons exhibit a

179 mixture of preparation-related, triggering-related, and execution-related activity. Yet a small percentage

180 of recorded neurons displayed nearly 'pure' preparatory activity, affording an opportunity to tentatively

181 explore some predictions. Consider the response illustrated in Figure 3a. For single-reach conditions, 182 delay-period activity is strongly selective: high before reaches to a down-and-left target and low before 183 reaches to a down-and-right target. Selectivity collapses by movement onset. This pattern held across all 184 conditions (Extended Data Fig. 4) but for simplicity is shown here for the two target locations that were 
bioRxiv preprint doi: https://doi.org/10.1101/2020.06.09.143040; this version posted June 10, 2020. The copyright holder for this preprint (which was not certified by peer review) is the author/funder, who has granted bioRxiv a license to display the preprint in perpetuity. It is made available under aCC-BY-NC-ND 4.0 International license.

\section{Fig. 3}

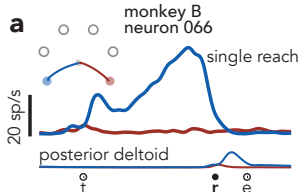

b
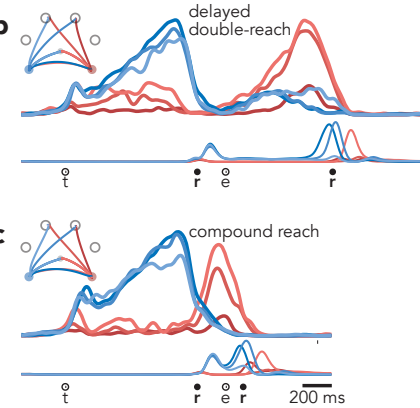
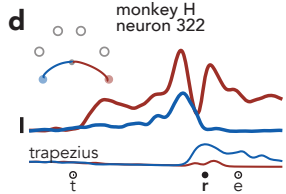

e

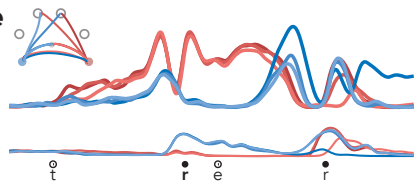

$f .20$

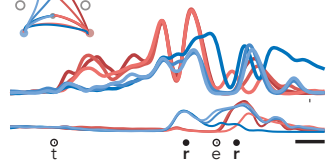

Fig. 3 | Responses of single neurons. a, Activity of one neuron, recorded from monkey B during single reaches to bottom-right (red) and bottom-left (blue) targets. Thin traces at bottom plot the activity of the posterior deltoid. Circles indicate the time of target onset $(t)$, reach onset $(r)$, and reach end (e). All traces are trial-averages of data that have been aligned twice: once to target onset and once to reach onset. See Methods for a complete description of data pre-processing. $\mathbf{b}$, Response of the same neuron and muscle during delayed double-reach conditions. Monkey B performed six such conditions, all of which are shown. $\mathbf{c}_{\text {, }}$ Response of the same neuron and muscle during compound reaches. Trace color corresponds to the direction of the first reach. Monkey B performed ten compound reach conditions, but for illustration only those with corresponding delayed double-reach conditions are shown. Data for all conditions is plotted in Extended Data Fig. 4. d, Activity of one neuron, recorded from monkey $\mathrm{H}$ during single reaches. Same format as $\mathbf{a}$. Thin traces at bottom plot the activity of the trapezius. e, Activity of the same neuron and muscle during delayed double-reaches (same format b). f, Activity of the same neuron and muscle during compound reaches. Responses during all tested conditions are plotted in Extended Data Fig. 5a. 
also used for the first reach of delayed double-reaches. (For monkey B, delayed double-reaches used fewer combinations than compound reaches, to allow different inter-reach pause durations while maintaining reasonable trial-counts.) The response of this example neuron is consistent with participation in preparatory computations but not execution-related computations. This interpretation is supported by responses during delayed double-reaches (Fig. 3b). Due to the imposition of a $600 \mathrm{~ms}$ pause between reaches, one expects monkeys to adopt a sequential strategy: the first reach should be prepared during the initial instructed delay, and the second should be prepared during the imposed pause. Consistent with that expectation, this neuron is selectively active during the initial instructed delay, largely silent during the first movement, and active again during the imposed pause (Fig. 3b). The second bout of (presumed) preparatory activity has different selectivity from the first, as expected given that the second reach differed from the first. In both cases, the pattern of putatively preparatory activity collapses as muscle activity starts changing (traces at bottom).

197 Compound reaches were executed so rapidly that the two epochs of muscle activity fused (Fig. 3c, bottom traces). However, the neuron's response did not shift to the pattern predicted by the holistic strategy. That hypothesis predicts that preparatory activity during the instructed delay should reflect the full two-reach movement, and thus differ from the preparatory activity before single reaches. This was

201 not observed. The three compound reaches that began with a down-and-left reach (Fig. 3c, blue traces)

202 shared similar firing rates during the instructed delay, despite differing second reaches. Indeed, these

203 firing rates were similar to that when preparing only a down-and-left reach (Fig. 3a). Analogously,

204 activity was low for the three compound reaches that began with a down-and-right reach (red traces), in 205 agreement with activity for a single down-and-right-reach. Furthermore, the neuron was active not only 206 before the first reach, but again before the onset of the second reach, suggesting a second bout of 207 preparation. More broadly, this neuron's response pattern during compound reaches (Fig. 3c) was 208 similar to that during delayed double-reaches (Fig. 3b), but with a much earlier second peak. During 209 compound movements, the second peak occurred during the first movement rather than during the 210 pause between movements. This neuron was recorded from monkey B, for whom delayed double211 reaches were also examined with imposed pauses of $300 \mathrm{~ms}$ and $100 \mathrm{~ms}$ (in addition to the $600 \mathrm{~ms}$ 212 pause in Fig. 3b). Inspection of this neuron's response across pause durations (Extended Data Fig. 4) 213 confirms that the compound-reach response lies on a continuum with the delayed-double reach 214 responses. Thus, the responses of this example neuron suggest that not only is the sequential strategy 215 employed during delayed double-reaches (as expected), it may continue to be employed during compound reaches. 
217 Of course, little can be concluded from the response of one neuron. Not only might that response not be

218 representative, it allows examination of only a subset of the key predictions. A challenge when

219 considering all neurons is that most are active during both preparation and movement. Such responses

220 are typically complex even during single reaches (Fig. 3d) and more so during delayed double-reaches

221 (Fig. 3e) and compound reaches (Fig. 3f). Indeed, once one considers all conditions (only a subset of

222 which are shown in Fig. 3) the responses of most neurons are bafflingly complex (Extended Data Fig. 5)

223 and it becomes nearly impossible to address simple questions - such as whether compound movements

224 involve two bouts of preparation - via inspection. Testing the competing predictions of the sequential

225 and holistic strategies thus depends upon separating preparatory and execution-related signals at the

226 population level.

Time-course of Preparatory Activity

228 Preparatory and execution-related signals were recently shown to be separable at the population-

229 level ${ }^{21,36}$. The correlation structure between neurons is essentially unrelated during preparation versus

230 movement. Thus, the neural dimensions that best capture preparatory activity are nearly orthogonal to

231 those that best capture execution-related activity. Preparatory and execution-related signals can

232 therefore be separated via projection onto appropriate sets of orthogonal dimensions. Identifying those

233 dimensions requires leveraging task epochs where preparation is occurring without execution and vice

234 versa. Our task affords a few such epochs. There is no movement during the instructed delay or the

235 pause between delayed double-reaches. Yet the pending reach is presumably being prepared at these

236 times. Conversely, movement execution is (by definition) occurring during single reaches, each delayed

237 double-reach, and the final reach of compound reaches - times when preparation is unlikely because

238 there is no immediately pending next movement. We used activity from these epochs to define 20

239 preparatory and 20 execution dimensions. This approach carries a caveat: dimensions optimized to

240 capture preparation before one set of reaches may sub-optimally capture preparation before different

241 reaches. As an abstract example, dimensions optimized to capture preparation for slow reaches are

242 expected to imperfectly capture preparation for fast reaches. This matters because the second

243 compound reach is driven by modestly different patterns of muscle activity (see above) from the

244 corresponding second delayed double-reach. Thus, any preparation for the second compound reach

245 may be incompletely captured by the preparatory dimensions, as they were optimized to capture 
preparation for modestly different movements. This limitation is acceptable so long as one is conservative when interpreting measures of variance captured.

Projecting population data onto the first preparatory dimension (Fig. 4a-c) yields a response similar to a single-neuron PSTH (with greater symmetry due to mean-centering during preprocessing, see Methods).

250 This resemblance is expected: the activity patterns captured by these dimensions are building blocks of

251 single-neuron responses. For example, the first preparatory dimension was the primary contributor

252 (with a large negative weight) to the response of the example neuron in Fig. 3a-c, accounting for $92 \%$ of

253 its across-condition response structure. Of course, this purity was rare; most neurons contained sizeable 254 contributions from multiple dimensions, both preparatory and execution-related. This highlights the 255 utility of the projections, which can be relatively pure even when single neurons are not.

256 During delayed double-reaches, activity in the first preparatory dimension is expected to obey the 257 predictions of the sequential strategy. This was indeed the case (Fig. 4b). There were two bouts of 258 strong selectivity: one before the first reach (peaking just before movement onset) and another before 259 the second reach (again peaking just before movement onset). Activity before the first reach was similar 260 to that before the corresponding single reach. Red traces cluster together and resemble the response 261 before a single down-and-right reach. Blue traces cluster together and resemble the response before a 262 single down-and-left reach. During the second bout of preparation, the order of the traces largely 263 inverts, in agreement with the physical reversal of the reach (when the first was rightwards, the second 264 had a leftwards component).

265 During compound reaches, activity in the first preparatory dimension (Fig. 4c) obeyed the predictions of 266 the sequential strategy and violated the predictions of the holistic strategy. There were two bouts of 267 preparatory subspace activity. The first occurred during the instructed delay. The second peaked just as 268 the first movement ended. Furthermore, there was no evidence that the first bout of preparation 269 reflected the two-reach sequence. The ten traces in Fig. 4c are color coded by the identity of the first 270 reach. The pattern of preparatory activity before the first reach was nearly identical to that before single 271 reaches (Fig 4a).

272 The full pattern of activity during compound reaches was very similar to that during delayed double273 reaches (which had four fewer conditions) with the exception of timing. For delayed double-reaches, the 274 second bout of preparation occurred when the hand was stationary during the imposed pause. For 275 compound reaches, the second bout of preparation occurred essentially contiguously with the decline of 
bioRxiv preprint doi: https://doi.org/10.1101/2020.06.09.143040; this version posted June 10, 2020. The copyright holder for this preprint (which was not certified by peer review) is the author/funder, who has granted bioRxiv a license to display the preprint in perpetuity. It is made available under aCC-BY-NC-ND 4.0 International license.

Fig. 4
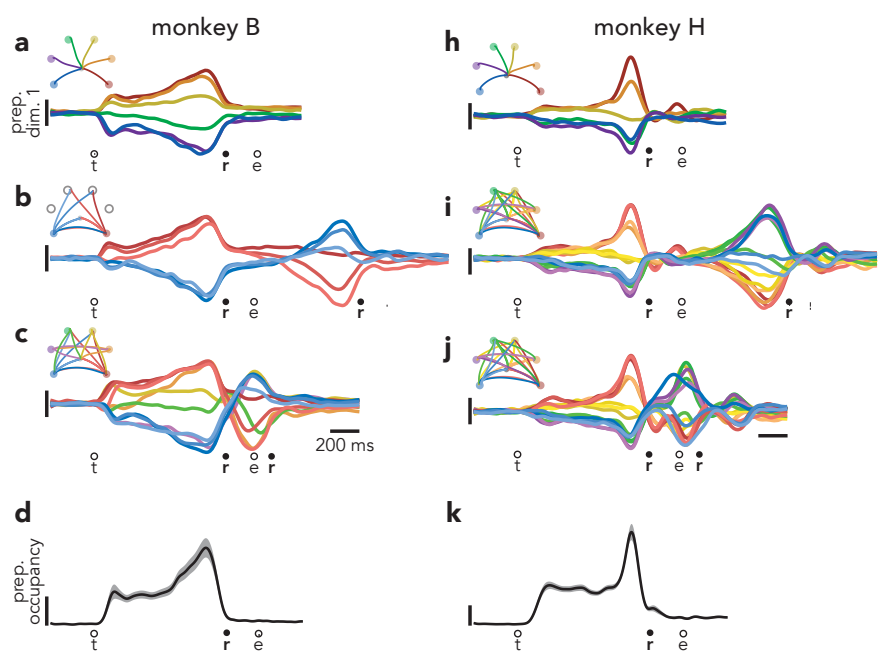

$\mathbf{k}$

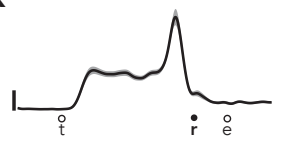

e

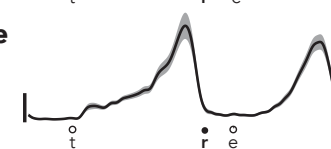

f
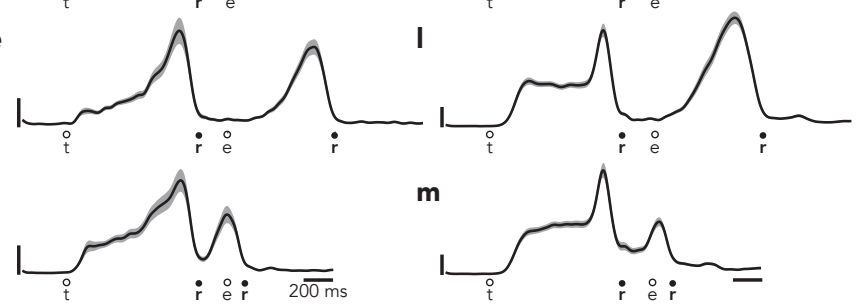

m
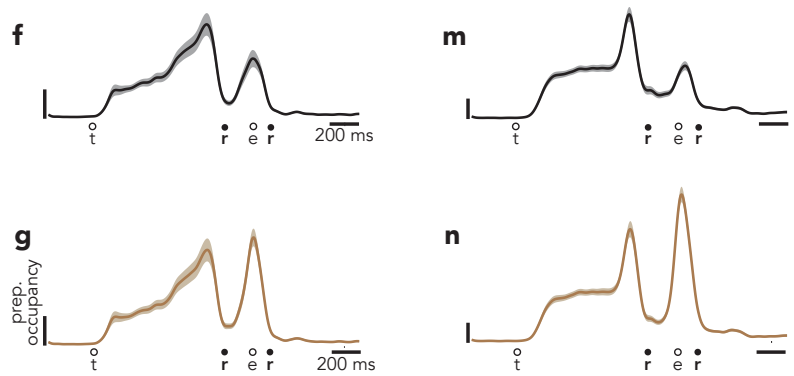

Fig. 4 | Time-course of activity in preparatory dimensions. a, Projection of population activity, during single reaches, onto the first preparatory dimension. Data are from monkey B. Unlike in Fig. 5, data are shown for all conditions. Vertical scaling is arbitrary and conserved across panels. $\mathbf{b}$, The same projection for delayed double-reaches. $\mathbf{c}$, The same projection for compound reaches. d, Preparatory subspace occupancy (for all 20 dimensions) during single reach conditions. Shaded regions indicate the standard deviation of the sampling error (equivalent to the standard error of the mean) estimated by resampling. As occupancy is a measure of normalized firing rates, the units are arbitrary. e, Preparatory subspace occupancy during delayed double-reaches. f, Preparatory subspace occupancy during compound reaches. $\mathbf{g}$, Same as $\mathbf{f}$, but preparatory dimensions were found using an expanded range of times that included activity during the brief dwell-period between compound reaches. $\mathbf{h}$-j, Same as a-c but for monkey $\mathrm{H}$. Note that monkey $\mathrm{H}$ performed more two-reach conditions than monkey B. k-n, Same as $\mathbf{d}-\mathbf{g}$ but for monkey H. Occupancy just prior to the second reach in compound reach conditions $(\mathbf{f}, \mathbf{m})$ was significantly greater than the occupancy during the same time period of single reaches for both monkeys ( $p<0.001$ for both monkeys via a bootstrap procedure; see Methods). 
the first bout. Thus, the second bout of preparatory dimension activity was developing while the hand

277 was still in flight. Because monkey B performed delayed double-reaches with different instructed

278 pauses, we could ask whether activity during compound reaches lay on a continuum with those

279 conditions. In other words, is the pattern of activity similar, but with the second bout of preparation

280 simply occurring earlier? This was indeed the case (Extended Data Fig. 6). Thus, activity in the first

281 preparatory dimension was consistent with the hypothesis that, during compound reaches, only the first

282 reach is prepared during the instructed delay period and the second reach is then prepared as the hand

283 is in flight.

284 To assess activity across all preparatory dimensions, we computed preparatory subspace occupancy: the across-condition variance of the neural state as a function of time. For single-reach conditions (Fig. 4d) the preparatory subspace was occupied during the instructed delay but not during the reach ${ }^{21,38}$. For delayed double-reaches (Fig. 4e; Extended Data Fig. 6g,h,i), the preparatory subspace was occupied twice, once before each reach. This continued to be true for compound reaches (Fig. 4f), consistent with the results observed above for the first preparatory dimension. For compound reaches, the second peak was smaller than the first. This might seem to suggest that the second bout of preparation is weaker. However, a smaller second peak is expected for technical reasons. As discussed above, dimensions

292 optimized to capture preparation before one set of movements are essentially guaranteed to sub293 optimally capture preparation for a different set of movements. To be conservative, activity before the

294 second compound reach was not used when optimizing preparatory dimensions (we wished to 295 determine whether a second bout of preparation naturally emerged). If one is less conservative, and

296 allows optimization to also employ the epoch just before the second compound reach, the two 297 preparatory peaks are of comparable size (Fig. 4g, Extended Data Fig. 7).

298 Results were similar for monkey H (Fig. 4, right column). Monkey H performed a larger set of two-reach 299 combinations, all of which were performed both as delayed double-reaches and compound reaches. To 300 prevent trial-counts from becoming overly high, the instructed pause was always 600 ms for delayed 301 double-reaches. Otherwise the task was unchanged. Results were correspondingly similar. Compound 302 reaches involved two bouts of preparation (Fig. 4j,m,n). During the first bout, selectivity matched that 303 before single reaches and before delayed double-reaches (compare order of colored traces across 304 panels h-j). 
The analysis above indicates that delay-period activity before compound reaches is similar to that before corresponding single reaches. To explore further, we chose a time-point near the end of the initial bout of preparation: 120 ms before first-reach onset. In the top two preparatory dimensions, the pattern of neural states resembled the spatial layout of the reach targets (Fig. 5a,b). This is true even though preparatory activity is not a literal representation of target location ${ }^{23}$. For compound reaches (circles)

311 preparatory states clustered according to first-reach identity. For example, blue circles correspond to 312 three compound reaches that all begin with a down-and-left reach. These cluster near the state before a 313 single down-and-left reach (blue triangle), despite involving dissimilar second reaches.

314 Across the top five preparatory dimensions and all conditions, the location of the preparatory state 315 before a compound reach was always similar to that before the corresponding single reach (Fig. 5c,d; $\rho=$ 3160.96 for both monkeys; $p<0.0001$ for both). We focused on the top five dimensions because each of the 317 additional fifteen captured, on its own, little variance. Nevertheless, results were nearly identical when 318 extended to all twenty preparatory dimensions: $\rho=0.92$ and 0.92 for both monkeys $(p<0.0001$ for 319 both).

320 According to our working paradigm, similar preparatory states should lead to similar time-evolving 321 patterns of execution-related activity. To test this, we repeated the above analysis for the execution322 related dimensions and for multiple times (Extended Data Fig. 8). Execution-related activity was very 323 similar for the first compound reach and the corresponding single reach: $\rho=0.91$ and 0.93 (monkey B 324 and $\mathrm{H}, \mathrm{p}<0.0001$ for both). Thus, the first reach of a compound reach involves preparatory and 325 execution-related neural events very similar to those accompanying a matched single reach.

326 A prediction of the sequential hypothesis is that not only should compound reaches involve two bouts of 327 preparation, but the second bout should reflect the second reach much as if it were performed in 328 isolation. An ideal test of this prediction is not possible because the second reach of a compound reach 329 is not identical to the second reach of a delayed double-reach; the former is generated while muscle 330 activity from the first reach is still dissipating. Yet while not identical, muscle activity patterns are still 331 fairly similar. The sequential hypothesis thus predicts a considerable similarity between the preparatory 332 state preceding the second compound versus delayed double-reach. This was indeed the case: $\rho=0.96$ 333 and 0.90 (monkeys $\mathrm{B}$ and $\mathrm{H}, \mathrm{p}<0.0001$, both monkeys). Notably, this was true despite the very different 334 circumstances under which preparatory state was measured: while the first compound reach was in 335 flight versus at the end of the imposed pause between delayed double-reaches. 
bioRxiv preprint doi: https://doi.org/10.1101/2020.06.09.143040; this version posted June 10, 2020. The copyright holder for this preprint (which was not certified by peer review) is the author/funder, who has granted bioRxiv a license to display the preprint in perpetuity. It is made available under aCC-BY-NC-ND 4.0 International license.

\section{Fig. 5}

a

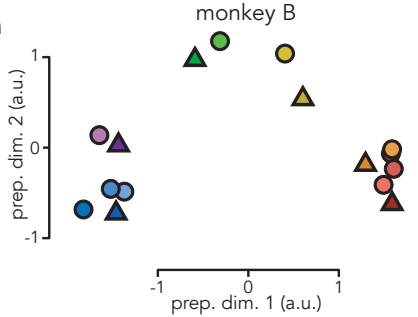

c

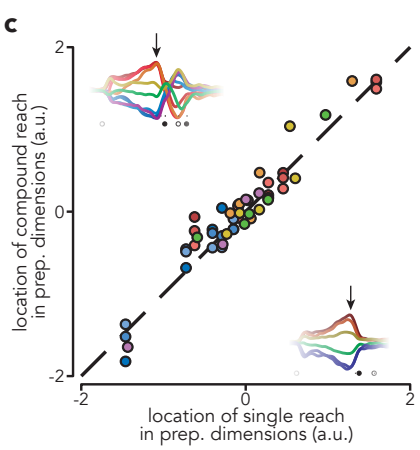

e

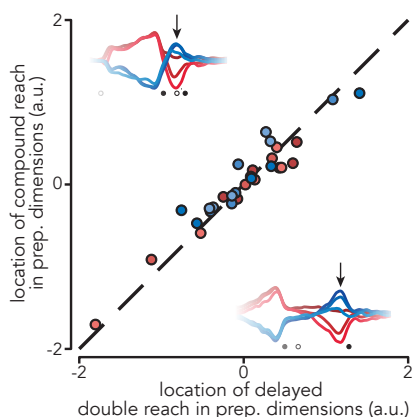

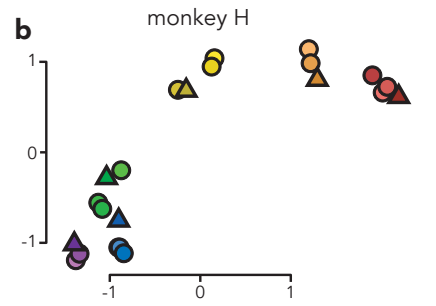

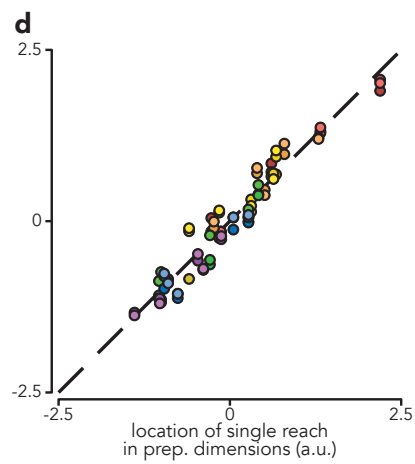

$\mathbf{f}$

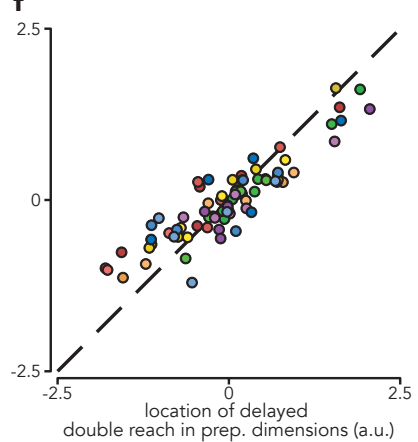

Fig. 5 | Patterns of preparatory activity. a, Projections of population activity, just before first-reach onset, onto the top two preparatory dimensions. Triangles represent single reach conditions and circles represent compound reach conditions. Marker color indicates first-reach direction. Data are from monkey B. b, Same as a but for monkey H. Monkey H performed more compound reach conditions than monkey B. c, Comparison of preparatory activity, before first-reach onset, between compound and single reaches. Each circle plots the location in preparatory subspace of activity before one compound reach versus that for the corresponding single-reach. Arrows on each inset indicate when preparatory subspace activity was assessed. Each compound-reach condition contributes five data-points - one for each of the top five preparatory dimensions. Dashed line indicates unity slope. Data are for monkey B. d, same as $\mathbf{c}$ but for Monkey H. e, Comparison of preparatory activity, prior to the second reach, between compound reaches and matched delayed double-reaches. Arrows on each inset indicate when preparatory subspace activity was assessed. f, same as e but for monkey $\mathrm{H}$. 
337 The final prediction of the holistic and sequential hypotheses concerns trigger-related events. We have 338 proposed that reaches are generated by a movement-specific preparatory neural state interacting with execution-related dynamics. Yet movement-specific signals, while prevalent, are not the largest signals in motor cortex during reaching. Instead, the dominant aspect of the neural response is independent of

341 reach identity. This 'condition invariant signal' (CIS) begins $\sim 150$ ms before movement onset ${ }^{27,28,39,40}$ and

342 is proposed to reflect a large input that triggers movement execution. Consistent with this, the

343 dominant response component in mouse motor cortex during reaching depends upon thalamic inputs, 344 and silencing those inputs interrupts execution ${ }^{39,41}$. Furthermore, the CIS is highly predictive of the exact 345 moment of reach initiation ${ }^{27}$. A similar CIS is observed in recurrent networks trained to produce reach346 related muscle activity ${ }^{37}$. In such networks, the CIS relates mechanistically to movement triggering: it 347 moves the neural state to a region with strong local dynamics. Working within this paradigm, the holistic 348 and sequential hypotheses make clear predictions. There should be a monophasic CIS if compound reaches are generated holistically and a biphasic CIS if they are generated sequentially.

The CIS occurs in dimensions nearly orthogonal to those capturing reach-specific activity ${ }^{27,28}$. To isolate 351 the CIS, we employed demixed principal component analysis ${ }^{45,46}$ to identify dimensions where activity 352 was time-varying but largely independent of reach direction ${ }^{27}$. There were two such condition353 independent $(\mathrm{Cl})$ dimensions ( $88 \%$ and $82 \%$ condition-invariant structure, monkey $\mathrm{B}$ and $\mathrm{H}$ ). We begin 354 by considering the projection onto one such dimension (Fig 6., top sub-panels). Consistent with prior 355 findings, during single reaches there was a large change in the neural state beginning 150 ms before movement onset and peaking around movement onset (Fig. 6a,d). There were two peaks during delayed double-reaches (Fig. 6b,e) and also during compound reaches (Fig. 6c,f).

358 Prior studies have found at least two $\mathrm{Cl}$ dimensions during reaching ${ }^{27,28}$. Yet visualization has typically 359 concentrated on one dimension, either plotted versus time or as part of a three-dimensional state space 360 where the other two dimensions capture condition-specific structure. Because we wish to concentrate on predictions regarding the $\mathrm{CIS}$, we ignore that condition-specific structure and plot neural activity in a

362 state-space spanned by two $\mathrm{Cl}$ dimensions (bottom of panels in Fig. 6). To aid visualization, we plot a 363 single condition-averaged trajectory, with colored portions indicating peri-reach times. Activity in the CIS 364 dimension returns, after a reach, close to its location before the reach and thus traces out a loop (Fig. $3656 a, d)$. We stress that this loop should not be interpreted as related to the dynamics that describe condition-specific activity, which possess a rotational component ${ }^{22,37}$. Condition-specific dynamics occur 
bioRxiv preprint doi: https://doi.org/10.1101/2020.06.09.143040; this version posted June 10, 2020. The copyright holder for this preprint (which was not certified by peer review) is the author/funder, who has granted bioRxiv a license to display the preprint in perpetuity. It is made available under aCC-BY-NC-ND 4.0 International license.

\section{Fig. 6}

a

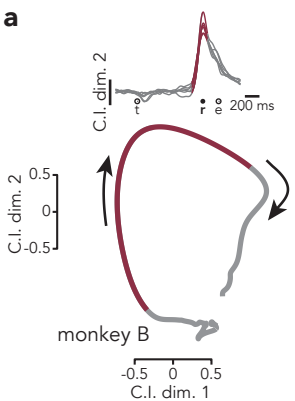

b

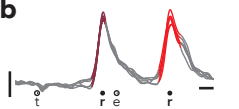

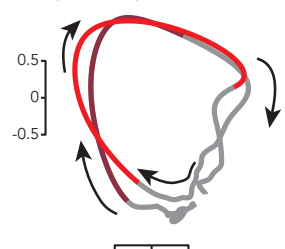

$\begin{array}{rrr}-0.5 & 0 & 0.5\end{array}$

e

d

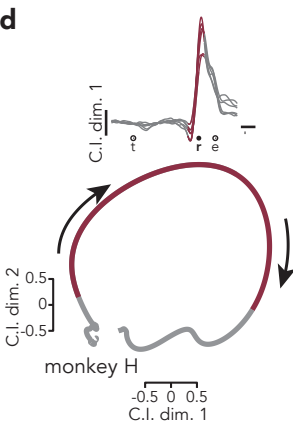

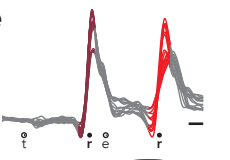

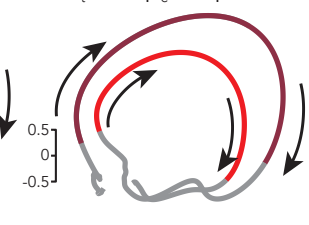

$-0.500 .5$
C

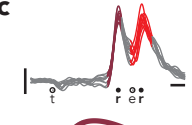

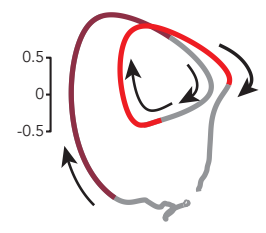

$\begin{array}{lll}-0.5 & 0 & 0.5\end{array}$

f

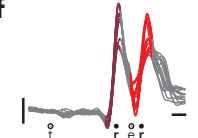

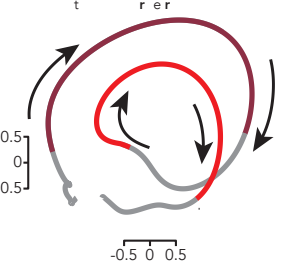

Fig. 6 | Evolution of the condition-invariant signal. a, Top: Projections of population activity from all single-reach conditions onto one condition-invariant dimension (the second, which best captured the rapid rise). The colored region of each trace highlights peri-reach activity (150 ms prior reach onset to $150 \mathrm{~ms}$ prior to reach end). Bottom: State-space projections of the same activity onto two condition-invariant dimensions. Because the projection is similar for all conditions, the average is shown to simplify presentation. As above, trace color highlights peri-reach activity. Arrows indicate the temporal evolution of activity. $\mathbf{b}$, Same as a but for delayed double-reaches. The two colored regions indicate peri-reach activity for the two reaches. $\mathbf{c}$, Same as b but for compound reaches. d-f, Same as a-c but for monkey H. 
367 in orthogonal dimensions ${ }^{27,28}$ and describe the different trajectories across many conditions. The looping

368 trajectory in Figure 6 is similar across all conditions and is thus not evidence for a flow-field with a

369 rotational component (indeed in models it is produced by an input rather than by network dynamics).

370 During single reaches (Fig. 6a,d) the looping CIS trajectory was traversed once. There were two loops

371 during delayed double-reaches (Fig 6b,e). Because there was time for the trajectory to relax to baseline

372 between reaches, the second loop simply began from the same region as the first. During compound

373 reaches, the trajectory also displayed two loops (Fig 6c,f), with the second loop beginning before the

374 first had fully relaxed. This timing lay on a continuum with that observed for delayed double-reaches

375 with different pause durations (Extended Data Fig. 9). Thus, the CIS displays the structure predicted by

376 the sequential hypothesis during both delayed double-reaches and during compound reaches.

\section{An RNN that generates compound reaches}

378 The empirical results above universally agree with the predictions of the sequential hypothesis. We

379 found this surprising. Not only did we expect that motor cortex would holistically generate compound

380 reaches, it seemed implausible that it could do otherwise. Given the rapid pace of execution, the

381 sequential strategy requires that preparation for the second reach occur during execution of the first.

382 That overlap is not necessarily surprising in and of itself. It is known that preparatory activity can overlap

383 execution-related activity, both as movements are initiated (the former wanes as the latter declines ${ }^{21}$ )

384 and during corrections ${ }^{38}$. However, in all such cases the purpose of preparatory activity is proposed to

385 be a near-immediate impact on execution-related activity. This is consistent with the proposal that a

386 mechanistic purpose of preparatory activity is to seed execution-related activity ${ }^{22,23,47}$. How can

387 preparatory activity do so for the second reach without disrupting the ongoing first reach?

388 To address this question, we trained a recurrent neural network to produce patterns of reach-related

389 muscle activity (Fig. 7). As in Sussillo et al. ${ }^{37}$, the network received two input types. The first was a three-

390 dimensional preparatory input that specified reach identity (light gray traces). The second was a

391 condition-independent 'go cue' that indicated when the reach should occur (dark gray trace). The

392 network then had to produce the target muscle-activity pattern for that reach (purple traces). We

393 trained our network to generate two reaches on each trial, driven by two bouts of preparatory inputs

394 and two go cues. The separation between go cues was variable and could be relatively long (Fig. 7a) or 
bioRxiv preprint doi: https://doi.org/10.1101/2020.06.09.143040; this version posted June 10, 2020. The copyright holder for this preprint (which was not certified by peer review) is the author/funder, who has granted bioRxiv a license to display the preprint in perpetuity. It is made available under aCC-BY-NC-ND 4.0 International license.

\section{Fig. 7}

a

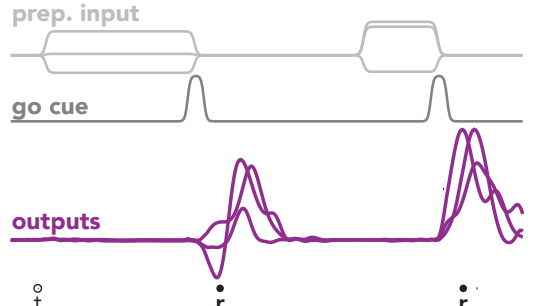

b

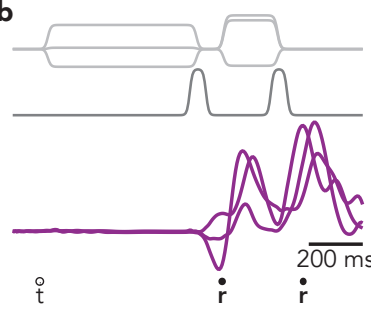

e
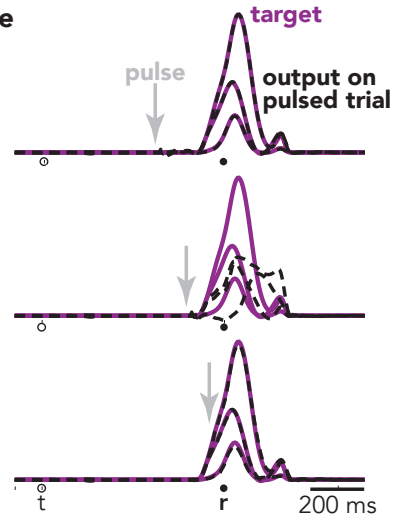

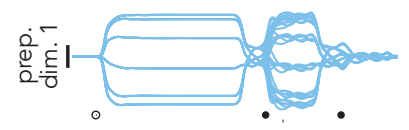

d

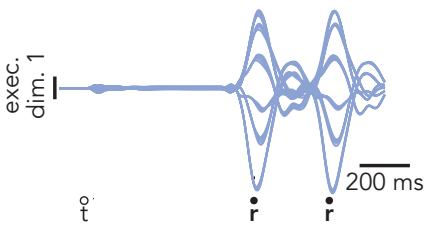

f

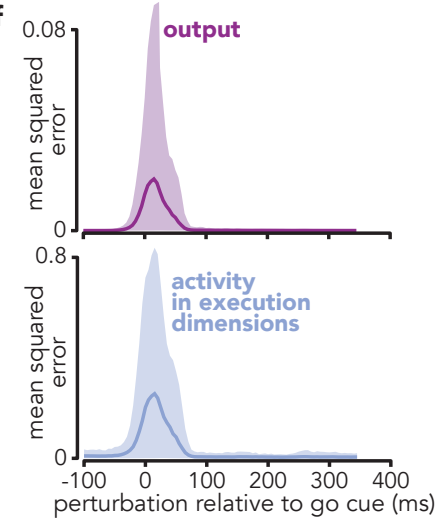

Fig. 7 | A recurrent network trained to generate reach sequences. a, On each trial, the network was required to produce two 'blocks' of output (purple traces), each consisting of the empirical activity of six muscles (only three of which are plotted for clarity) for one reach.

Muscle activity was recorded from monkey B. The network received both a three-dimensional condition-specific 'preparatory' input (light gray traces) and one-dimensional condition-invariant a go cue (dark gray trace). On this example trial, the network was required to produce two blocks of output (two 'reaches') with a long pause between. b, Example trial where the inputs required the network to produce two blocks of output with no pause between, analogous to generating a compound reach. $\mathbf{c}$, Projections of network activity onto the first preparatory dimension. Each trace plots activity for one of 36 compound reach conditions. Preparatory and execution dimensions were found using the same method applied to the neural data. $\mathbf{d}$, Projections of network activity onto the first execution dimension. e, Impact of perturbing pulses, delivered to the inputs that normally convey preparatory signals. Each subpanel plots the target (purple) and actual (black dashed) output. Three (of six) output dimensions are shown for simplicity. Arrows indicate the time when the perturbation was delivered. Same time-scale as $\mathbf{a}$ and $\mathbf{b}$. $\mathbf{f}$, Top: summary of the impact of disruptive pulses on network output as a function of perturbation time. Mean squared error (between normal and actual network output) was calculated over a $200 \mathrm{~ms}$ window beginning at perturbation onset. Shaded region indicates the range into which $95 \%$ of effects fell. Bottom: same but for the impact on activity in the execution dimensions. 
as short as $300 \mathrm{~ms}$ (Fig. 7b). In the latter case, the second set of preparatory inputs arrived while the network was still generating muscle activity for the first reach.

397 Our choice of inputs required the network to employ the sequential strategy - the network did not

398 'know' what the second reach would be until the arrival of the second preparatory input. We expected 399 this scenario might be challenging and that the network might fail or adopt an idiosyncratic strategy. In 400 fact, the network readily produced the target patterns of muscle activity across the full range of go cue 401 separations $\left(R^{2}>0.99\right)$. This ability did not depend upon a fundamentally different strategy for brief go 402 cue separations (see below). This implies that the network was able to absorb the second set of 403 preparatory inputs, setting the stage for the second reach, without disrupting ongoing execution of the

404 first reach. Plotting projections of network activity onto preparatory and execution dimensions (Fig.

$4057 c, d)$ revealed that the second bout of preparatory activity did indeed overlap with execution-related

406 activity for the first reach. Despite that overlap, the first-reach output was generated just as it would 407 have been without a second reach.

408 To determine how the network accomplished this, we probed the link between preparation-related 409 dimensions and network output. Because preparatory dimensions are 'muscle null', their influence on 410 the output occurs via the rest of the network and is thus amenable to modulation. To explore that 411 modulation, we employed brief input pulses that transiently perturbed activity in the preparatory

412 dimensions. This allowed us to probe at what times, relative to the go cue, preparatory activity

413 influences network output. Modulation was strong and temporally specific. Perturbations that coincided 414 with the go cue disrupted network output (Fig. 7e, middle) while earlier or later perturbations had no 415 effect. As a result, the influence of preparatory activity on network output was restricted to a narrow 416 window around the time of the go cue (Fig. 7f). Critically, that influence was negligible by $~ 80$ ms after 417 the go cue (Fig. 7f, top), as was the influence on execution-related dimensions (Fig. 7f, bottom). Thus, 418 not only is preparatory activity muscle-null, it is 'dynamically null' except during a brief window around 419 the go cue. This allowed the network to develop preparatory activity appropriate for the second reach 420 while muscle activity for the first reach was still being generated. The second bout of preparatory 421 activity had an impact only at the appropriate time: after the second go cue was given.

\section{Comparison of neural and network strategies}


423 Many of the basic features of the empirical data were replicated by the network model. Like empirical

424 neurons (Fig. 8a), model units exhibited complex responses that were typically a mixture of preparation-

425 related and execution-related activity (Fig. 8b). Despite such mixing at the single-unit level, preparatory

426 and execution-related activity in the model occupied orthogonal subspaces, making it possible to plot

427 projections that captured nearly pure preparatory (Fig. 7c) or execution-related (Fig. 7d) activity. Such

428 orthogonality is noteworthy because it is a consistent feature of empirical data, but is not typically

429 observed in networks trained to produce only single reaches ${ }^{36,37}$.

430 The orthogonality of subspaces makes it possible to compute preparatory- and movement-subspace

431 occupancy, just as we had for the data. Doing so reveals that, for both model and data, the sequential

432 strategy was employed both when there was considerable temporal separation between reaches (Fig.

$4338 \mathrm{a}, \mathrm{b}$ ) and when reaches occurred in rapid succession (Fig. 8c,d). For both model and data, a given reach

434 involved a stereotyped set of events: preparatory-subspace occupancy increased, the CIS 'triggering'

435 event occurred, and execution occupancy immediately followed. Sequences of two reaches simply

436 involved this set of events occurring twice. When the second reach occurred immediately after the first,

437 the second bout of preparation was briefer and overlapped execution of the first reach. 
bioRxiv preprint doi: https://doi.org/10.1101/2020.06.09.143040; this version posted June 10, 2020. The copyright holder for this preprint (which was not certified by peer review) is the author/funder, who has granted bioRxiv a license to display the preprint in perpetuity. It is made available under aCC-BY-NC-ND 4.0 International license.

\section{Fig. 8}

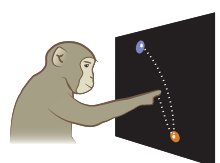

b
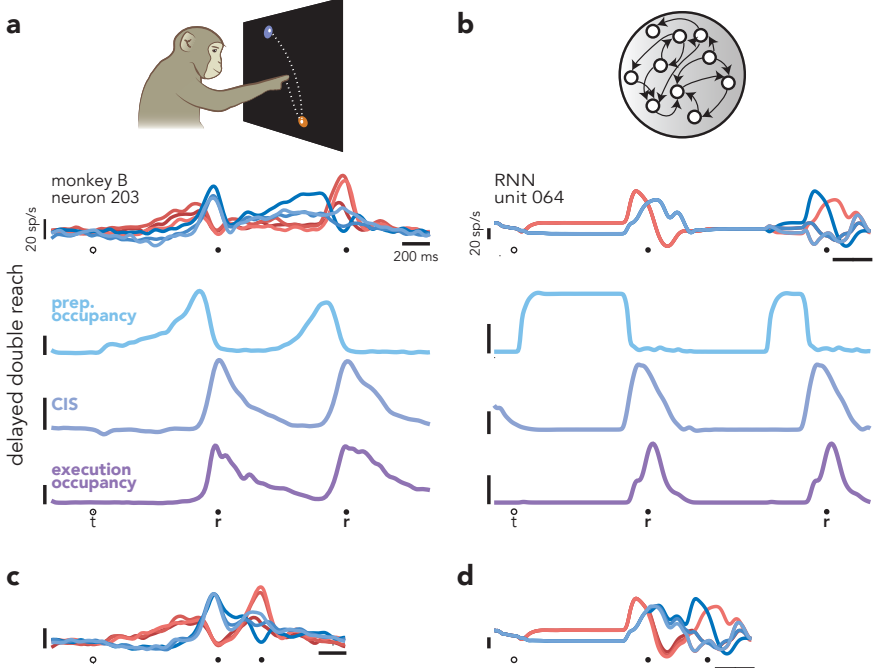

d
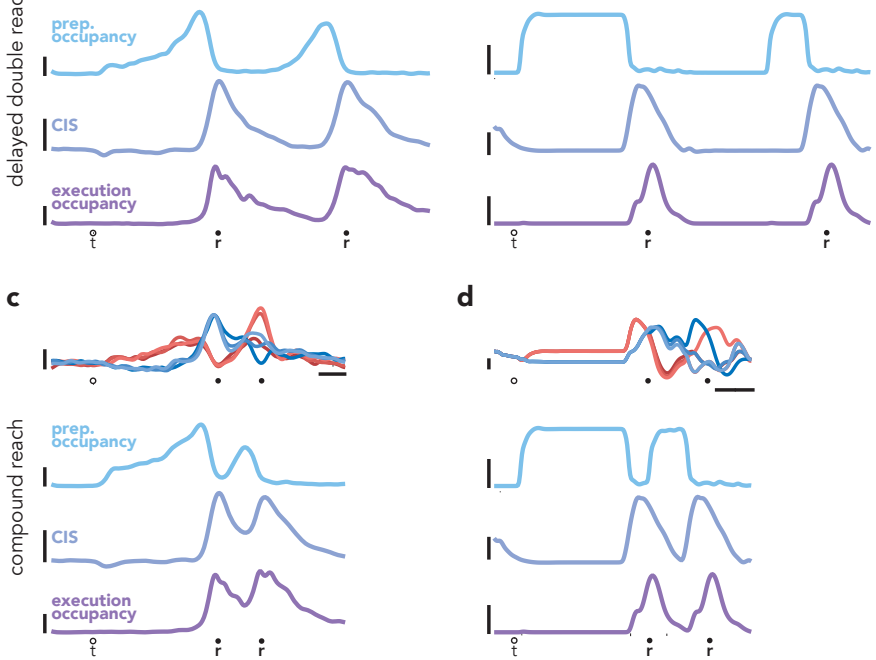

Fig. 8 | Similarities between motor cortical activity and network activity. a, Summary of motor cortical activity during delayed double-reach conditions. Although preparatory-, triggering-, and execution-related signals are mixed at the level of single neurons (top), within the population, these signals occur in orthogonal dimensions. Prior to each reach, preparatory dimensions become occupied first (light blue trace), then preparatory occupancy falls $\sim 150 \mathrm{~ms}$ before reach onset. At approximately the same time, a condition-invariant signal (violet trace) occurs and occupancy of execution dimensions (dark purple trace) increases. This same pattern is repeated prior to the second reach. Vertical scale is arbitrary but is preserved in panel c. $\mathbf{b}$, A similar sequence of events occurred in the network, which was trained to produce the empirical patterns of muscle activity from monkey $B$. The most notable difference between motor cortex and the network is the temporal envelope of preparatory occupancy. For the network, this results from the envelope of the network inputs; a more realistic pattern could be produced simply by altering that envelope, with essentially no change in network output. c, Motor cortex empirically generated compound reaches by simply repeating the sequence of events that generated delayed double-reaches. However, the second instance of the prepare-trigger-execute motif occurred shortly after the first. $\mathbf{d}$, As was true in the empirical data, the network produced compound reaches using the same sequence of computations that produced delayed double-reaches. 


\section{Discussion}

Motor sequences have been studied for over one hundred years ${ }^{48-50}$, yet it has remained unclear how

441 low-level computations contribute to generating sequential behavior. Does producing a rapidly

442 unfolding motor sequence require individual elements to be fused into larger, cohesive units? Or can motor cortex leverage a unified strategy to generate sequences at different paces?

444 In our task, compound reaches potentially appeared to be holistically generated at the level of the 445 muscles: muscle activity formed one continuous pattern spanning the full compound reach.

446 Nevertheless, motor cortical activity revealed that component reaches were prepared and executed

447 sequentially. Compound reaches involved two epochs of preparatory activity, each of which reflected

448 the identity of the immediately pending reach rather than the full sequence. Compound reaches also

449 involved two peaks of the condition-invariant signal, which is hypothesized to reflect movement

450 triggering. Execution-subspace activity reflected the reach being currently generated rather than the full

451 sequence - e.g., it was very similar during the first reach regardless of the presence or identity of the

452 next reach.

453 The ability to separate preparation-related and execution-related activity was central to many of the

454 above findings and also revealed how the rapid pace of compound reaches was achieved. That pace 455 depended not on the preparation of multiple reaches prior to initiation, but upon simultaneously 456 engaging preparation and execution. That temporal overlap allowed the second reach to be prepared 457 while the first was still unfolding. Related forms of overlap have been recently observed: preparatory 458 and execution-subspace activity overlap around movement onset ${ }^{21}$ and during externally driven 459 corrections ${ }^{38}$. However, in all such cases the presumed goal of preparatory-subspace activity was to 460 immediately impact motor output. We were initially surprised that preparatory subspace activity could 461 develop without impacting either execution-subspace activity or motor output. However, a neural 462 network trained to generate sequential reaches readily utilized this strategy. In doing so, it adopted 463 internal activity that displayed the empirically observed features described above (e.g., two bouts of 464 preparation, triggering, and execution).

465 We stress that our results do not prove that it is impossible to holistically generate a multi-element movement. Our goal was not to determine whether the holistic strategy is possible, but whether a 
holistic strategy is essential to the rapid production of motor sequences. Based on our findings, it is clear that a holistic strategy is not necessary for motor cortex to generate rapid sequences, nor does it emerge from familiarity with a particular sequence ${ }^{3,15,29}$. Our monkeys had extensive practice with compound movements and performed them so rapidly that muscle activity merged into a continuous

471 pattern, yet the sequential strategy was employed at the level of motor cortex. Nevertheless, it is quite

472 possible that a holistic strategy could be encouraged by specific training approaches, perhaps by training

473 on compound reaches alone (but never their component reaches) or by restricting sensory cues ${ }^{51}$. As

474 will be discussed below, a trivial version of the holistic strategy -- replacing two movements with one

475 that achieves a similar goal - is almost certainly possible.

477 The prepare-trigger-execute paradigm was developed in the context of single reaches and how they 478 could be generated by networks with strong internal dynamics. Whether this paradigm is applicable to 479 motor sequences is test of the framework's utility: do the data agree with self-consistent sets of predictions made by competing hypotheses developed within that paradigm? This was indeed the case, and it is worth emphasizing that this did not have to be true. The data could have agreed with some predictions of one hypothesis and some of the other, or with neither. Mixed or difficult-to-interpret results would have indicated that the paradigm needed to be reevaluated. Instead, in every case the data obeyed the predictions of the sequential strategy and not the holistic strategy. These results serve not only to support one hypothesis and reject the other, but also to demonstrate the utility of the paradigm used to couch the hypotheses and generate the predictions.

487 Our results also help extend the prepare-trigger-execute paradigm. Preparation has historically been considered a time-consuming process ${ }^{47,52-55}$ and thus to contribute to the longer time between sequence elements at chunk boundaries ${ }^{56,57}$. This made it reasonable to assume that minimizing temporal

490 separation required a holistic strategy. However, it has now been demonstrated that preparation can be 491 remarkably swift for well-practiced movements ${ }^{21,58}$. The present results confirm that swift preparation is 492 possible, allowing rapid sequences to be produced via the sequential strategy.

493 Our results also agree with recent findings showing or suggesting that preparatory activity can occur 494 during movement. Ames et al. ${ }^{38}$ found that preparatory dimensions become occupied during movement 495 when a jumping target required an immediate correction, and Stavisky et al. ${ }^{59}$ found that the response 
to an unexpected visual perturbation occurs first in an output-null space before flowing into an outputpotent space. The present study is the first to show that the preparatory subspace can be occupied in anticipation of a pending movement without causing a change in the present movement. This presumably depends upon the orthogonality of preparation and execution-related dimensions. Such orthogonality naturally appeared when we pushed networks at a pace that required preparation and execution to overlap. In contrast, the networks in $^{37}$ were never trained to perform reach sequences and did not show orthogonal preparation and execution. Thus, the need to develop preparatory activity despite ongoing movement provides a possible explanation for a consistent feature of the empirical population response. Additionally, the orthogonality of preparatory and execution dimensions may relate to optimal control of preparatory activity ${ }^{60}$.

A consequence of the sequential strategy is that, in caudal PMd and M1, activity before sequence onset reflects only the first reach. Yet upstream areas must presumably look further ahead. Previous work demonstrates that delay-period activity in the supplementary motor area (SMA) reflects the identity of

511 human subjects performing a key-press task; while there is no evidence for sequence-specific selectivity 512 in the M1 BOLD signal, such selectivity is present in upstream areas ${ }^{16-18}$. Using a cycling task, which

513 shares some features with sequence tasks, Russo et al. ${ }^{63}$ found that M1 activity reflects only the present 514 motor output while SMA activity distinguishes situations that will have different future outputs. When 515 we began this study, a reasonable hypothesis was that SMA coordinates sequences - e.g., specifies 516 which elements will be produced in what order ${ }^{64}$ - allowing motor cortex to generate closely spaced 517 elements holistically. Given present results, upstream computations must instead enable the sequential 518 strategy, which requires carefully timed preparatory and triggering inputs. These inputs likely originate not only from SMA but from many areas, both cortical ${ }^{62,65-67}$ and subcortical ${ }^{68-70}$.

520 Although skillfully and rapidly performing a sequence of motor elements does not depend on a holistic 521 strategy at the level of motor cortex, there may still be cases where skilled action depends on replacing 522 elements - i.e., learning new movements ${ }^{4}$. For example, when learning to sign their name, a person 523 transitions from writing each letter deliberately to producing the entire signature in fewer, smoother 524 strokes. Of course, the shape of individual letters changes throughout this process, as do the patterns of 525 muscle activity that produce them. There is thus an important distinction between learning to more 
526 rapidly link elements (but preserving the elements themselves) and replacing one set of actions with an

527 entirely new one that serves a similar high-level goal but is otherwise a different movement ${ }^{64,71}$. The

528 former can be driven by a sequential strategy (with greater skill perhaps resulting from more rapid and

529 consistent pacing of the different stages) while latter requires the holistic strategy by definition.

\section{Acknowledgments}

532 We thank Y. Pavlova for excellent animal care. This work was supported by the Grossman Center for the

533 Statistics of Mind, the Simons Foundation (M.M.C.), the McKnight Foundation (M.M.C.), NIH Director's

534 New Innovator Award DP2 NS083037 (M.M.C.), NIH CRCNS R01NS100066 (M.M.C.), NIH 1U19NS104649

535 (M.M.C.), P30 EY019007 (M.M.C), the National Science Foundation (A.J.Z.), and the Kavli Foundation

536 (M.M.C.), 
Subjects were two adult, male macaque monkeys (monkeys $\mathrm{B}$ and $\mathrm{H}$ ). Animal protocols were approved by the Columbia University Institutional Animal Care and Use Committee (AC-AAAM2550). Experiments were controlled and data collected under computer control (Speedgoat Real-time Target Machine). During experiments, monkeys sat in a customized chair with the head restrained via a surgical implant.

544 Stimuli were displayed on an LCD monitor in front of the monkey, and a tube dispensed juice rewards.

545 The left arm was comfortably restrained, and the task was performed with the right arm.

Hand position was monitored using an infrared optical system (Polaris; Northern Digital) to track ( 0.3 $\mathrm{mm}$ precision) a reflective bead temporarily affixed to the third and fourth digits. Each trial began when the monkey touched and held a central touch-point. After holding for 400-600 ms (randomized) targets appeared - one for single reach conditions and two for delayed double-reach and compound reach conditions. There were six possible target locations, arranged radially $140 \mathrm{~mm}$ from the touch-point. Targets were round and had to be hit with within $25 \mathrm{~mm}$ of their center. A condition was defined by target location(s) and whether success required a single reach, a delayed double-reach, or a compound reach. Conditions were interleaved using a block-randomized design.

554 For single reach conditions, a $20 \mathrm{~mm}$ diameter, green target appeared at one of the six locations. After a 555 variable delay period (0-1000 ms), the target doubled in size and the central touch point disappeared.

556 These events served as salient go cue, instructing the monkey to reach. Reaches were successful if they 557 were initiated 100-500 ms after the go cue, lasted $<500 \mathrm{~ms}$, and stayed on the target for $600 \mathrm{~ms}$ with 558 minimal hand motion. These requirements were conserved across all conditions. For compound reach conditions, two targets appeared simultaneously. Target color indicated the first

560 (green) and second (blue) targets. After a variable delay period (0-1000 ms) both targets grew in size 561 and the touch point disappeared, providing the go cue. The monkey then captured the first target and 562 was immediately given a juice reward. After capturing the second target (and holding for $600 \mathrm{~ms}$ ) the 563 monkey was given a second juice reward. During compound reach conditions, we encouraged the 564 monkey capture the second target as quickly as possible after capturing the first. We used a speed 565 threshold (70 mm/sec, roughly $5 \%$ of peak velocity) to determine when the first target was captured. As 566 soon as hand velocity fell below this threshold, the second target began to shrink rapidly, such that it 
disappeared (and the trial was aborted) after 350 ms. Shrinking stopped when the second reach began,

568 providing incentive to do so quickly. In practice, monkeys reached considerably faster than required, with a median pause between reaches of 119 ms (monkey G) and 137 ms (monkey H).

570 Delayed double-reach conditions started as for compound reaches, with a key exception. The first target 571 was colored only in its center - the rest was white. The diameter of the colored center indicated the 572 duration of the imposed pause between reaches. Diameters of $8 \mathrm{~mm}, 14 \mathrm{~mm}$, and $18 \mathrm{~mm}$ indicated 573 pauses of $600 \mathrm{~ms}, 300 \mathrm{~ms}$, and $100 \mathrm{~ms}$. The imposed pause began when the hand reached the first 574 target. At that moment, the colored portion of the target began to grow at a constant rate. The 575 monkey's hand was required to remain within the target with minimal movement until the colored 576 center reached the perimeter of the first target (i.e., the target was all green). Immediately after

577 becoming all green, the first target disappeared and the second target instantly grew in size, indicating 578 that the monkey was now permitted to reach towards the second target. A successful second reach 579 required initiation within $500 \mathrm{~ms}$ ( $<100 \mathrm{~ms}$ reaction times were allowed here, as the time of the second go-cue was predictable) and then holding the final target for $600 \mathrm{~ms}$. Reward was delivered after both the end of the pause between reaches and at the end of the final hold period.

\section{Neural and Muscle Recordings}

After initial training, we performed sterile surgery to implant a head restraint and cylindrical recording chamber, which was positioned to give access to the arm area of dorsal premotor cortex (PMd) and primary motor cortex (M1). Recordings for both monkeys were performed in the left hemisphere. The task was performed with the right arm. Chamber positioning was guided by structural magnetic resonance imaging prior to implantation. We used intra-cortical microstimulation to confirm that our recordings were from the forelimb region of motor cortex (biphasic pulses, cathodal leading, $250 \mathrm{~ms}$ pulse width delivered at $333 \mathrm{~Hz}$ for a total duration of $50 \mathrm{~ms}$ ). Microstimulation typically evoked contractions of the shoulder and upper-arm muscles, with current thresholds between $20 \mu \mathrm{A}-200 \mu \mathrm{A}$,

592 recorded in both M1 and PMd and from a variety of depths). We recorded single-neuron responses 593 using one or more 32-channel linear-array electrodes (S-probes; Plexon) lowered into cortex using a 594 motorized microdrive. Spikes were either sorted offline by hand (Plexon Offline Sorter) or automatically 595 using KiloSort ${ }^{72}$. Spike clusters found by KiloSort were manually curated (Phy Template-gui software; 596 Kwik Team). We recorded all well-isolated task-responsive neurons; no attempt was made to screen any 
response property. Spikes were smoothed with a Gaussian kernel with standard deviation of $25 \mathrm{~ms}$ and

598 averaged across trials to produce peri-stimulus time histograms. Across all recorded neurons, the average minimum trial-count per condition was $\sim 20$ trials.

600 In dedicated sessions, we recorded electromyogram (EMG) activity using intramuscular electrodes from 601 the following muscles: trapezius, anterior, lateral, and posterior heads of the deltoid, lateral head of the 602 biceps, pectoralis, and brachialis. EMG signals were bandpass filtered (50-5kHz), digitized at $30 \mathrm{kHz}$,

603 rectified, smoothed with a Gaussian kernel with standard deviation $25 \mathrm{~ms}$, and averaged across trials to 604 produce a continuous estimate of muscle activity intensity.

\section{Data Pre-Processing}

606 Photodetectors (Thorlabs) were used to synchronize commands from the behavioral control software 607 with the $60 \mathrm{~Hz}$ refresh rate of the display, such that the timing of visual events was known with $1 \mathrm{~ms}$ 608 accuracy. Hand position was sampled at $60 \mathrm{~Hz}$. For analysis on a one millisecond timescale, values were 609 interpolated and filtered. For analysis, movement onset and offset were estimated offline based on 610 hand speed. Movement onset (for each reach) was defined as the time that hand speed first surpassed $6115 \%$ of the peak speed for that reach. Similarly, movement end was defined as the time when hand speed 612 first dropped below $5 \%$ of the peak reach speed.

613 In order to provide a clear view of preparatory activity during the instructed delay period, all analyses 614 only included trials with instructed delays $>500 \mathrm{~ms}$. To provide a continuous view of neural activity 615 during the delay period, data were aligned to target onset and the onset of the first reach and 616 concatenated. Concatenation occurred $350 \mathrm{~ms}$ prior to the onset of the first reach.

617 To compare the temporal evolution of neural activity across time and conditions, we aligned single trials

618 to key behavioral events. For each set of conditions that shared a temporal task structure, we scaled the 619 time-base for each trial such that the duration of the first reach and the dwell-time between reaches 620 matched the median first reach duration and dwell-time for that set of conditions. This was done 621 separately for single reaches, compound reaches, and delayed double-reaches. Monkey B performed 622 delayed double-reaches with three different pause durations; the above procedure was performed 623 separately for each. All variables of interest (firing rate, hand speed, EMG) were computed for each trial 624 prior to alignment. Thus, alignment never alters the magnitude of these variables, it simply modifies 625 slightly when they occur. Using an alternative alignment procedure that did not involve rescaling - 
626 aligning single-trial data to target onset, first reach onset, and second reach onset and concatenating -

627 produced very similar results.

\section{Determining Sequence Selectivity}

629 We defined sequence selectivity in the traditional way: responses that were statistically different when

630 a reach was part of a sequence versus performed alone. First reaches provided the most natural

631 comparison, as they were physically similar when performed alone versus as part of a sequence. We

632 thus asked whether activity before and during first reaches differed between the single-reach and

633 compound-reach conditions. We computed the average firing rate on each trial during two epochs: the

634 delay period and an 'execution-epoch' spanning the first reach. A t-test (unpaired) was used to ask

635 whether, for each epoch, these differed for single versus compound reaches. For the execution epoch,

636 we employed a second comparison that avoids the concern that differences might be missed when

637 averaging across time-points. We compared, at each millisecond, firing-rates for single versus compound

638 reaches, and took the average absolute difference. This was summed across conditions (so that we

639 made one comparison per neuron). We used a resampling test to determine if the summed difference

640 was greater than expected given trial-to-trial variability. Resampling was performed by pooling all single

641 and compound reach trials, and drawing two new 'conditions' (sampling with replacement). Differences

642 were considered significant at the 0.001 level if the true difference was greater than 1000 resampled

643 differences.

\section{Identifying Preparatory and Execution Dimensions}

645 As in previous work ${ }^{21,22}$ we employed two additional preprocessing steps prior to dimensionality

646 reduction. First, we soft-normalized each neuron's firing rate. The normalization factor was equal to that

647 neuron's firing rate range plus five spikes/s. This encouraged dimensionality reduction to capture the

648 responses of all neurons, rather than just high firing-rate neurons. Second, within each set of conditions

649 of the same duration (e.g., single reaches, compound reaches) we mean-centered the firing rate of each

650 neuron, so that its average rate (across conditions) was zero at all times. This step ensures that

651 dimensionality reduction focuses on dimensions where activity is selective across conditions.

652 Dimensions capturing condition-invariant activity ${ }^{27,28,39,40}$ were identified separately (see below). We

653 employed a recently developed dimensionality reduction approach ${ }^{21,36,38}$, which leverages the fact that

654 motor cortex population activity occupies nearly orthogonal subspaces during preparation and 
655

Using activity from the above times, we constructed two matrices: $P \in R^{N x} T_{p r e p}$ which holds the neural

677 activity from preparatory epochs and $E \in R^{N x} T_{\text {exec }}$ which holds activity from the execution epochs. $N$ is

678 the number of recorded neurons, $T_{\text {prep }}$ is the total number of times across all conditions and all

679 preparatory epochs as defined above, and $T_{\text {exec }}$ is the total number of times across all conditions and

execution ${ }^{36}$. Importantly, this remains true even for preparatory events that occur outside an instructed delay period ${ }^{21,38}$. Identifying those dimensions requires two sets of data: one where activity reflects only preparation and another where activity reflects only execution.

Preparation-only activity was obtained using task epochs where the hand was stationary but preparation was presumed to occur. Most basically this included a 'delay-period' epoch from $700-150$ ms prior to the onset of single reaches. We similarly employed the delay-period epoch before compound reaches and delayed double-reaches. For the delayed double-reaches, we used trials with a $600 \mathrm{~ms}$ inter-reach pause (which was experienced by both monkeys). These same trials provided an additional 'secondreach preparatory' epoch: 300 ms -150 ms prior to the onset of the second reach. We included this epoch because second reaches had trajectories that were different from first reaches (they moved from one peripheral target to the other, rather than from the center out) and we wished preparatory dimensions to capture preparation for both first and second reaches. To be conservative, we did not use a second-reach epoch before compound reaches; one of the competing hypotheses predicted no preparatory activity at that time, and we wished to avoid any concern that our method might 'build it in.'

Execution-only activity was obtained from a peri-reach epoch, -50-500 ms relative to reach onset. We used data only for reaches where it was unlikely that preparation overlapped execution. This included single reaches, both reaches for delayed double-reaches with a $600 \mathrm{~ms}$ pause (the longest pause we used), and the second reach of compound reaches. For all these reaches, any subsequent reach (either second reaches or the return to the center) is some distance in the future, such that any preparation for the next movement likely occurs after completion of the present reach.

all execution epochs as defined above. We sought a set of preparatory dimensions $W_{\text {prep }}$ that maximally capture the variance in $P$, and an orthogonal set of dimensions $W_{\text {exec }}$ that maximally capture the variance of $E$. To do so, we computed covariance matrices $C_{p r e p}=\operatorname{cov}(P)$ and $C_{\text {exec }}=\operatorname{cov}(E)$ and used numerical optimization to find: 


$$
\left[W_{\text {prep }}, W_{\text {exec }}\right]=\operatorname{argmax}_{\left[W_{\text {prep }}, W_{\text {exec }}\right]} \frac{1}{2}\left(\frac{\operatorname{Tr}\left(W_{\text {prep }}^{T} C_{\text {prep }} W_{\text {prep }}\right)}{\sum_{i=1}^{d_{\text {prep }}} \sigma_{\text {prep }}(i)}+\frac{\operatorname{Tr}\left(W_{\text {exec }}^{T} C_{\text {exec }} W_{\text {exec }}\right)}{\sum_{i=1}^{d_{\text {exec }}} \sigma_{\text {exec }}(i)}\right)
$$

$$
\text { subject to: } W_{\text {prep }}^{T} W_{\text {exec }}=0, W_{\text {prep }}^{T} W_{\text {prep }}=I, W_{\text {exec }}^{T} W_{\text {exec }}=I
$$

686

687

688

689

690

691

692

693

694

695

696

697

698

699

700

701

702

703

704

705

706

707

708

where $\sigma_{\text {prep }}(i)$ is the $i^{\text {th }}$ singular value of $C_{\text {prep }}$ and $\sigma_{\text {exec }}(i)$ is the $i^{\text {th }}$ singular value of $C_{\text {exec. }} . \operatorname{Tr}(\cdot)$ is the matrix trace operator. $\operatorname{Tr}\left(W_{\text {prep }}^{T} C_{\text {prep }} W_{\text {prep }}\right)$ is the variance captured, across all preparatory epochs, by the preparatory dimensions. $\operatorname{Tr}\left(W_{\text {exec }}^{T} C_{\text {exec }} W_{\text {exec }}\right)$ is the variance captured, across all execution epochs, by the execution dimensions. The optimization objective is normalized (by the singular values) to make it insensitive to differences in total preparatory versus execution variance. Using a dimensionality of 20, $W_{\text {prep }}$ and $W_{\text {exec }}$ captured $78 \%$ and $68 \%$ of the variance in all preparatory epochs for monkey $\mathrm{B}$ and monkey $\mathrm{H}$, respectively and $68 \%$ and $70 \%$ of the variance in all execution epochs for monkey $\mathrm{B}$ and monkey $\mathrm{H}$, respectively.

Some analyses, in particular those involving visualization, involved focusing on a subset of the dimensions within $W_{\text {prep }}$. To do so, we found $W_{\text {prep_rotate }}$, a set of dimensions spanning the same subspace but ordered so that the first dimension captures the most variance and so forth (as for PCA). To avoid this rotation being biased towards one type of reach, it was based on preparatory epoch activity from an equal number of times before first versus second reaches. This encouraged the first two dimensions to capture preparation-related activity before both first and second reaches (it did not ensure equal variance for both, but encouraged parity that if indeed preparatory activity was of similar magnitude for both). To find $W_{\text {prep_rotate }}$, we projected a $200 \mathrm{~ms}$ window of activity before all six single reaches (starting $300 \mathrm{~ms}$ before reach onset) and a $200 \mathrm{~ms}$ window of activity before six delayed double-

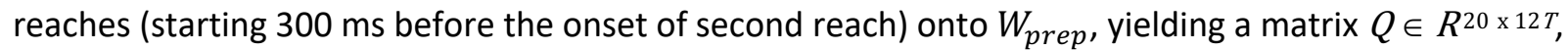
where $T$ was equal to 200 . We performed PCA on $Q$, yielding a rotation matrix $B \in R^{20 \times 20}$. $W_{\text {prep_rotate }}$ was then $W_{\text {prep }} B$. All population analyses were performed by projecting population data onto $W_{\text {prep_rotate }}$. This rotation of the basis set was important when focusing on a subset of dimensions (one wishes to prioritize variance captured) but had no impact on analyses that employed all dimensions (e.g., $W_{\text {prep }}$ and $W_{\text {prep_rotate }}$ capture the same total variance). 
710 We calculated occupancy as described in ${ }^{21,36}$. Below we describe how we computed preparatory

711 occupancy. Execution occupancy was computed analogously. For a given time $t$ and condition $\Phi$, the

712 projection of the population response onto the preparatory dimensions is: $x^{p r e p}(t, \phi)=$

$713 W_{\text {prep_rotate }} \boldsymbol{r}(t, \phi)$, where $\boldsymbol{r}(t, \phi)$ is a vector containing response of each neuron for time $t$ and

714 condition $\phi$. To measure subspace occupancy, we calculated:

$$
\operatorname{occupancy}_{\text {prep }}(t)=\sum_{k=1}^{20} \operatorname{var}_{\phi}\left(x_{k}^{\text {prep }}(t, \phi)\right)
$$

716 Where $\operatorname{var}_{\phi}$ indicates taking the variance across conditions and $x_{k}^{\text {prep }}$ is the $k^{\text {th }}$ element of $x^{\text {prep }}$. We

717 employed a resampling procedure to estimate sampling error. We created 1000 surrogate neural

718 populations by redrawing, with replacement, neurons from the original population. For each, we

719 computed the preparatory dimensions and subspace occupancy, yielding a distribution of occupancies.

720 That distribution was used to compute error bars and for assessing statistical significance. For example,

721 it allowed us to ask whether preparatory subspace occupancy during the first compound reach was

722 significantly higher than that during single reaches.

723 To compare preparatory occupancies between single reaches and compound reaches, we asked within a

724 particular window (200 ms window ending with the onset of the second reach), how frequently (across

725 surrogate populations) was preparatory occupancy higher for compound reaches than for single

726 reaches. If compound reach occupancy was higher in $95 \%$ of comparisons, this would yield a p-value of

$727 \quad 0.05$

729 We used $\mathrm{dPCA}^{45,46}$ to identify neural dimensions where activity varies primarily with time and not 730 condition $^{27}$. dPCA was applied to a matrix of firing rates, $A \in R^{C T X N}$, where $N$ is the number of neurons, $731 C$ is the number of conditions, and $T$ is the number of time-points per condition. The data in $A$ was taken 732 from a $300 \mathrm{~ms}$ window centered on reach onset. Data was included for all of the reaches from single-, 733 compound-, and delayed double-reach-conditions - i.e., all reach directions and reach types. dPCA 734 leverages lables assigned to each row of $A$ that identify the condition and time. dPCA seeks a matrix $735 W_{d P C A} \in R^{N X k}$, where $k$ is specified (we chose $k$ to be 8 ), that produces a projection $X=A W_{d P C A}$. Each 736 column of $W_{d P C A}$ is a dimension and each column of $X$ is thus a projection of the population response 
737

738

739

740

741

742

743

744

onto that dimension. Like PCA, dPCA optimizes dimensions to capture variance, such that $A \approx X W_{d P C A}^{T}$. Unlike PCA, dPCA further optimizes $W_{d P C A}$ such that each column of $X$ covaries strongly with only one label (i.e., time or condition). Our analysis of the condition-invariant signal employed those columns where activity varied primarily with time. We observed very similar results if compound reach conditions were omitted when finding $W_{d P C A}$.

\section{Training a Recurrent Neural Network to Generate Sequences of EMG}

To determine whether a recurrent neural network (RNN) could readily use the sequential strategy, we trained an RNN to reproduce the empirical patterns of muscle activity, largely following the procedure outlined in ${ }^{37}$. We used a network with dynamics:

$$
\boldsymbol{x}(t+1, c)=f\left(A \boldsymbol{x}(t, c)+B \boldsymbol{u}(c)+\boldsymbol{b}_{\text {recurrent }}+\boldsymbol{w}(t, c)\right)
$$

where $\boldsymbol{x}$ is the network state for time $t$ and condition $c$. The function $f$ was a hyperbolic tangent linking each unit's inputs to its firing rate, $A \boldsymbol{x}$ captures the influence of network activity on itself via connection weights $A, B \boldsymbol{u}$ represents the external inputs, $b_{\text {recurrent }}$ is a vector of offset biases, and the random vector $\boldsymbol{W} \sim N\left(0, \sigma_{W} I\right)$ adds a small amount of noise. We set $\sigma_{W}$ to be $5 \times 10^{-4}$. Network output was a linear readout of $\boldsymbol{X}$ :

$$
\boldsymbol{y}(t, c)=C \boldsymbol{x}(t, c)+\boldsymbol{b}_{\text {readout }}
$$

The parameters $A, B, C, \boldsymbol{b}_{\text {recurrent }}$, and $\boldsymbol{b}_{\text {readout }}$ were optimized to minimize a loss function composed of the difference between the network output, $y$ and a target, $\boldsymbol{y}_{\text {targ }}$ and a number of regularization terms (see below). The target output was based upon EMG recorded from six muscles from monkey B during all six individual reach conditions. We created 36 'conditions' - each a two-reach combination that the network had to perform. Each condition was generated by concatenating muscle activity from two reaches, separated by an interval where the target output was 0 . On each 'trial' the network performed one condition, with a random value of that interval $(0-600 \mathrm{~ms})$. Thus, the model was asked to produce six-dimensional muscle activity for all 36 possible two-reach combinations, and to do so across a wide range of delays.

The loss function optimized during training was:

$$
L=\sum_{t=1, c=1}^{T, C}\left[\frac{1}{2}\left\|\boldsymbol{y}_{\mathrm{targ}}(t, c)-\boldsymbol{y}(t, c)\right\|_{2}^{2}\right]+\frac{\lambda_{A}}{2}\|A\|_{F}^{2}+\frac{\lambda_{C}}{2}\|C\|_{F}^{2}+\sum_{t=1, c=1}^{T, C}\left[\frac{\lambda_{x}}{2}\|\boldsymbol{x}(t, c)\|_{2}^{2}\right]+\frac{\lambda_{J}}{2} R_{J}
$$


764 The first term is the error between the network output and the target. All other terms serve to

765 regularize the network solution. The second and third terms penalize large recurrent and output

766 weights, respectively. The fourth term penalizes large firing rates, and the final term discourages

767 complex dynamics 37 .

$$
R_{J}=\frac{1}{C T} \sum_{t=1, c=1}^{T, C}\left\|\frac{\partial(A f(\boldsymbol{x}(t, c)))}{\partial(\boldsymbol{x}(t, c))}\right\|_{F}^{2}
$$

769 This final term was not necessary for the network to successfully learn to produce the target output, nor

770 the general solution it found (i.e. placing preparatory, triggering, and execution activity in largely

771 orthogonal sets of dimensions). However, as $\mathrm{in}^{37}$, regularization in general and the inclusion of $R_{J}$ in

772 particular encouraged network solutions that resembled, at the population and single-neuron level,

773 those observed empirically. Regularization coefficients were chosen to be: $\lambda_{A}=10^{-7}, \lambda_{C}=10^{-7}, \lambda_{x}=10^{-}$

7748 , and $\lambda_{J}=5 \times 10^{-5}$. The RNN was composed of 100 units, received 3 condition-specific inputs, and a

775 single condition-independent 'go-cue'. In order to ensure that the network was truly producing an

776 output in response to the go-cue (and not implicitly time-locking to the start of simulation) we used a

777 variable delay between condition-specific input onset and go-cue onset; this delay varied randomly

778 between 200 and $600 \mathrm{~ms}$. The matrices $A, B$, and $C$ were initialized as random orthonormal matrices,

779 and the network was trained using TensorFlow's Adam optimizer.

780 We asked how the network responded to disruptive pulses delivered around the time of the go-cue.

781 Each pulse was delivered via the network inputs for $30 \mathrm{~ms}$ and was a scaled version of the input vector

782 associated with one of the non-tested (i.e. off-target) reach directions. The magnitude of each pulse

783 vector was between 0.5 and 3 times that of the original input vector. Each time-point in Fig. $7 f$ was

784 probed with 1000 pulses. 


\section{References}

786

787

788

789

790

791

792

793

794

795

796

797

798

799

800

801

802

803

804

805

806

807

808

809

810

811

812

813

814

815

816

817

818

819

820

821

822

823

824

825

826

827

1 Sakai, K., Kitaguchi, K. \& Hikosaka, O. Chunking during human visuomotor sequence learning. Exp Brain Res 152, 229-242, doi:10.1007/s00221-003-1548-8 (2003).

2 Rosenbaum, D. A., Kenny, S. B. \& Derr, M. A. Hierarchical control of rapid movement sequences. Journal of experimental psychology. Human perception and performance 9, 86-102, doi:10.1037//0096-1523.9.1.86 (1983).

3 Abrahamse, E. L., Ruitenberg, M. F., de Kleine, E. \& Verwey, W. B. Control of automated behavior: insights from the discrete sequence production task. Front Hum Neurosci 7, 82, doi:10.3389/fnhum.2013.00082 (2013).

4 Ramkumar, P. et al. Chunking as the result of an efficiency computation trade-off. Nat Commun 7, 12176, doi:10.1038/ncomms12176 (2016).

5 Bo, J. \& Seidler, R. D. Visuospatial working memory capacity predicts the organization of acquired explicit motor sequences. J Neurophysiol 101, 3116-3125, doi:10.1152/jn.00006.2009 (2009).

6 Verwey, W. B. Effect of sequence length on the execution of familiar keying sequences: lasting segmentation and preparation? J Mot Behav 35, 343-354, doi:10.1080/00222890309603155 (2003).

7 Stadler, M. A. Implicit serial learning: questions inspired by Hebb (1961). Memory \& cognition 21, 819-827, doi:10.3758/bf03202749 (1993).

8 Verwey, W. B. \& Dronkert, Y. Practicing a Structured Continuous Key-Pressing Task: Motor Chunking or Rhythm Consolidation? J Mot Behav 28, 71-79, doi:10.1080/00222895.1996.9941735 (1996).

9 Verwey, W. B. Diminished motor skill development in elderly: indications for limited motor chunk use. Acta Psychol (Amst) 134, 206-214, doi:10.1016/j.actpsy.2010.02.001 (2010).

10 Ben-Shaul, Y. et al. Neuronal activity in motor cortical areas reflects the sequential context of movement. J Neurophysio/ 91, 1748-1762, doi:10.1152/jn.00957.2003 (2004).

11 Lu, X. \& Ashe, J. Anticipatory activity in primary motor cortex codes memorized movement sequences. Neuron 45, 967-973, doi:10.1016/j.neuron.2005.01.036 (2005).

12 Lu, X. \& Ashe, J. Dynamic reorganization of neural activity in motor cortex during new sequence production. Eur J Neurosci 42, 2172-2178, doi:10.1111/ejn.12979 (2015).

13 Kettner, R. E., Marcario, J. K. \& Port, N. L. Control of remembered reaching sequences in monkey. II. Storage and preparation before movement in motor and premotor cortex. Exp Brain Res 112, 347-358, doi:10.1007/bf00227941 (1996).

14 Hatsopoulos, N. G., Paninski, L. \& Donoghue, J. P. Sequential movement representations based on correlated neuronal activity. Exp Brain Res 149, 478-486, doi:10.1007/s00221003-1385-9 (2003).

15 Wymbs, N. F. \& Grafton, S. T. The Human Motor System Supports Sequence-Specific Representations over Multiple Training-Dependent Timescales. Cereb Cortex 25, 42134225, doi:10.1093/cercor/bhu144 (2015).

16 Yokoi, A., Arbuckle, S. A. \& Diedrichsen, J. The Role of Human Primary Motor Cortex in the Production of Skilled Finger Sequences. J Neurosci 38, 1430-1442, doi:10.1523/jneurosci.2798-17.2017 (2018). 
82817 Yokoi, A. \& Diedrichsen, J. Neural Organization of Hierarchical Motor Sequence Representations in the Human Neocortex. Neuron 103, 1178-1190.e1177, doi:10.1016/j.neuron.2019.06.017 (2019).

83118 Berlot, E., Popp, N. J. \& Diedrichsen, J. A critical re-evaluation of fMRI signatures of motor sequence learning. bioRxiv, 2020.2001.2008.899229, doi:10.1101/2020.01.08.899229 (2020).

834

19 Weinrich, M., Wise, S. P. \& Mauritz, K. H. A neurophysiological study of the premotor cortex in the rhesus monkey. Brain 107 ( Pt 2), 385-414, doi:10.1093/brain/107.2.385 (1984).

83720 Evarts, E. V. \& Tanji, J. Gating of motor cortex reflexes by prior instruction. Brain Res 71, 479-494, doi:0006-8993(74)90992-5 [pii] (1974).

83921 Lara, A. H., Elsayed, G. F., Zimnik, A. J., Cunningham, J. \& Churchland, M. M. Conservation of preparatory neural events in monkey motor cortex regardless of how movement is initiated. Elife 7, doi:10.7554/eLife.31826 (2018).

22 Churchland, M. M. et al. Neural population dynamics during reaching. Nature 487, 51-

56, doi:10.1038/nature11129 (2012). motor cortex reflects the speed of the upcoming reach. $J$ Neurophysiol 96, 3130-3146, doi:10.1152/jn.00307.2006 (2006).

25 Even-Chen, N., Sheffer, B., Vyas, S., Ryu, S. I. \& Shenoy, K. V. Structure and variability of delay activity in premotor cortex. PLoS Comput Biol 15, e1006808, doi:10.1371/journal.pcbi.1006808 (2019). Dynamics. Annu. Rev. Neurosci (2020). Kaufman, M. T. et al. The Largest Response Component in the Motor Cortex Reflects Movement Timing but Not Movement Type. eNeuro 3, doi:10.1523/ENEURO.008516.2016 (2016). Lara, A. H., Cunningham, J. P. \& Churchland, M. M. Different population dynamics in the supplementary motor area and motor cortex during reaching. Nat Commun 9, 2754, doi:10.1038/s41467-018-05146-z (2018). Matsuzaka, Y., Picard, N. \& Strick, P. L. Skill representation in the primary motor cortex after long-term practice. J Neurophysiol 97, 1819-1832, doi:10.1152/jn.00784.2006 (2007). sequences in monkey. I. Activity during movement in motor and premotor cortex. Exp Brain Res 112, 335-346, doi:10.1007/bf00227940 (1996).

31 Crammond, D. J. \& Kalaska, J. F. Prior information in motor and premotor cortex: activity during the delay period and effect on pre-movement activity. J Neurophysiol 84, 9861005 (2000). 
87032 Sergio, L. E. \& Kalaska, J. F. Changes in the temporal pattern of primary motor cortex

871

872

873

874

875

876

877

878

879

880

881

882

883

884

885

886

887

888

889

890

891

89240

893

894

895

896

897

898

899

900

901

902

903

904

905

906

907

908

909

$910 \quad 48$

911

$912 \quad 49$ activity in a directional isometric force versus limb movement task. J Neurophysiol 80, 1577-1583, doi:10.1152/jn.1998.80.3.1577 (1998).

33 Kalaska, J. F. \& Crammond, D. J. Cerebral cortical mechanisms of reaching movements. Science 255, 1517-1523, doi:10.1126/science.1549781 (1992).

34 Scott, S. H. \& Kalaska, J. F. Reaching movements with similar hand paths but different arm orientations. I. Activity of individual cells in motor cortex. J Neurophysiol 77, 826852 (1997).

35 Ames, K. C., Ryu, S. I. \& Shenoy, K. V. Neural dynamics of reaching following incorrect or absent motor preparation. Neuron 81, 438-451, doi:10.1016/j.neuron.2013.11.003 (2014).

36 Elsayed, G. F., Lara, A. H., Kaufman, M. T., Churchland, M. M. \& Cunningham, J. P. Reorganization between preparatory and movement population responses in motor cortex. Nat Commun 7, 13239, doi:10.1038/ncomms13239 (2016).

37 Sussillo, D., Churchland, M. M., Kaufman, M. T. \& Shenoy, K. V. A neural network that finds a naturalistic solution for the production of muscle activity. Nat Neurosci 18, 10251033, doi:10.1038/nn.4042 (2015).

38 Ames, K. C., Ryu, S. I. \& Shenoy, K. V. Simultaneous motor preparation and execution in a last-moment reach correction task. Nat Commun 10, 2718, doi:10.1038/s41467-01910772-2 (2019).

39 Dacre, J. et al. Cerebellar-recipient motor thalamus drives behavioral context-specific movement initiation. bioRxiv, 802124, doi:10.1101/802124 (2019).

40 Pani, P. et al. Neuronal population dynamics during motor plan cancellation in nonhuman primates. bioRxiv (2019).

41 Sauerbrei, B. A. et al. Cortical pattern generation during dexterous movement is inputdriven. Nature 577, 386-391, doi:10.1038/s41586-019-1869-9 (2020).

42 Gallego, J. A. et al. Cortical population activity within a preserved neural manifold underlies multiple motor behaviors. Nat Commun 9, 4233, doi:10.1038/s41467-01806560-z (2018).

43 Gallego, J. A., Perich, M. G., Chowdhury, R. H., Solla, S. A. \& Miller, L. E. Long-term stability of cortical population dynamics underlying consistent behavior. Nat Neurosci 23, 260-270, doi:10.1038/s41593-019-0555-4 (2020).

44 Sadtler, P. T. et al. Neural constraints on learning. Nature 512, 423-426, doi:10.1038/nature13665 (2014).

45 Kobak, D. et al. Demixed principal component analysis of neural population data. Elife 5, doi:10.7554/eLife.10989 (2016).

46 Brendel W, Romo R \& C, M. in Adv Neural Info Process Syst Vol. 24:1-9 (2011).

47 Churchland, M. M., Yu, B. M., Ryu, S. I., Santhanam, G. \& Shenoy, K. V. Neural variability in premotor cortex provides a signature of motor preparation. J Neurosci 26, 3697-3712 (2006).

48 Book, W. F. The psychology of skill: with special reference to its acquisition in typewriting. (University of Montana, 1908).

49 Miller, G. A., Galanter, E. \& Pribram, K. H. Plans and the structure of behavior. (1960). 
91350 Pew, R. W. Acquisition of hierarchical control over the temporal organization of a skill. J

$914 \quad$ Exp Psychol 71, 764 (1966).

91551 Ohbayashi, M., Picard, N. \& Strick, P. L. Inactivation of the Dorsal Premotor Area

916 Disrupts Internally Generated, But Not Visually Guided, Sequential Movements. J

$917 \quad$ Neurosci 36, 1971-1976, doi:10.1523/jneurosci.2356-15.2016 (2016).

91852 Rosenbaum, D. A. Human movement initiation: specification of arm, direction, and

$919 \quad$ extent. J Exp Psychol Gen 109, 444-474 (1980).

92053 Riehle, A. \& Requin, J. The predictive value for performance speed of preparatory

921

922

923 changes in neuronal activity of the monkey motor and premotor cortex. Behav Brain Res 53, 35-49, doi:10.1016/s0166-4328(05)80264-5 (1993).

924

925

54 Ghez, C. et al. Discrete and continuous planning of hand movements and isometric force trajectories. Exp Brain Res 115, 217-233 (1997).

55 Bastian, A., Schoner, G. \& Riehle, A. Preshaping and continuous evolution of motor cortical representations during movement preparation. Eur J Neurosci 18, 2047-2058 (2003).

Verwey, W. B. Buffer loading and chunking in sequential keypressing. Journal of Experimental Psychology: Human Perception and Performance 22, 544 (1996).

Verwey, W. B. Evidence for a multistage model of practice in a sequential movement task. Journal of Experimental Psychology: Human Perception and Performance 25, 1693 (1999).

Haith, A. M., Pakpoor, J. \& Krakauer, J. W. Independence of Movement Preparation and Movement Initiation. J Neurosci 36, 3007-3015, doi:10.1523/JNEUROSCI.3245-15.2016 (2016).

59 Stavisky, S. D., Kao, J. C., Ryu, S. I. \& Shenoy, K. V. Motor Cortical Visuomotor Feedback Activity Is Initially Isolated from Downstream Targets in Output-Null Neural State Space motor preparation: a thalamo-cortical circuit model. bioRxiv, 2020.2002.2002.931246, doi:10.1101/2020.02.02.931246 (2020).

61 Shanechi, M. M. et al. Neural population partitioning and a concurrent brain-machine interface for sequential motor function. Nat Neurosci 15, 1715-1722, doi:10.1038/nn.3250 (2012).

62 Tanji, J. \& Shima, K. Role for supplementary motor area cells in planning several areas. Annu Rev Neurosci 24, 631-651, doi:10.1146/annurev.neuro.24.1.631 (2001). movements ahead. Nature 371, 413-416, doi:10.1038/371413a0 (1994).

63 Russo, A. A. et al. Neural trajectories in the supplementary motor area and primary motor cortex exhibit distinct geometries, compatible with different classes of computation. bioRxiv, 650002, doi:10.1101/650002 (2019). prefrontal cortex reflects multiple steps of future events in action plans. Neuron 50, 631-641, doi:10.1016/j.neuron.2006.03.045 (2006). 
$95566 \quad$ Baldauf, D., Cui, H. \& Andersen, R. A. The posterior parietal cortex encodes in parallel

956 both goals for double-reach sequences. J Neurosci 28, 10081-10089,

957 doi:10.1523/jneurosci.3423-08.2008 (2008).

95867 Picard, N. \& Strick, P. L. Activation on the medial wall during remembered sequences of reaching movements in monkeys. J Neurophysiol 77, 2197-2201,

960 doi:10.1152/jn.1997.77.4.2197 (1997).

96168 Kornysheva, K. et al. Neural Competitive Queuing of Ordinal Structure Underlies Skilled Sequential Action. Neuron 101, 1166-1180.e1163, doi:10.1016/j.neuron.2019.01.018 (2019).

69 Jin, X., Tecuapetla, F. \& Costa, R. M. Basal ganglia subcircuits distinctively encode the parsing and concatenation of action sequences. Nat Neurosci 17, 423-430, doi:10.1038/nn.3632 (2014).

96971 Wong, A. L. \& Krakauer, J. W. Why Are Sequence Representations in Primary Motor

$970 \quad$ Cortex So Elusive? Neuron 103, 956-958, doi:10.1016/j.neuron.2019.09.011 (2019).

97172 Pachitariu, M., Steinmetz, N. A., Kadir, S. N., Carandini, M. \& Harris, K. D. in Advances in 972 neural information processing systems. 4448-4456. 


\section{Extended Data Fig. 1}

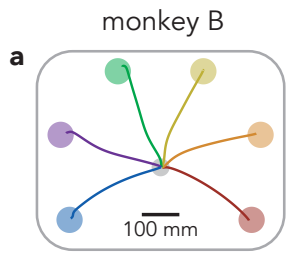

b
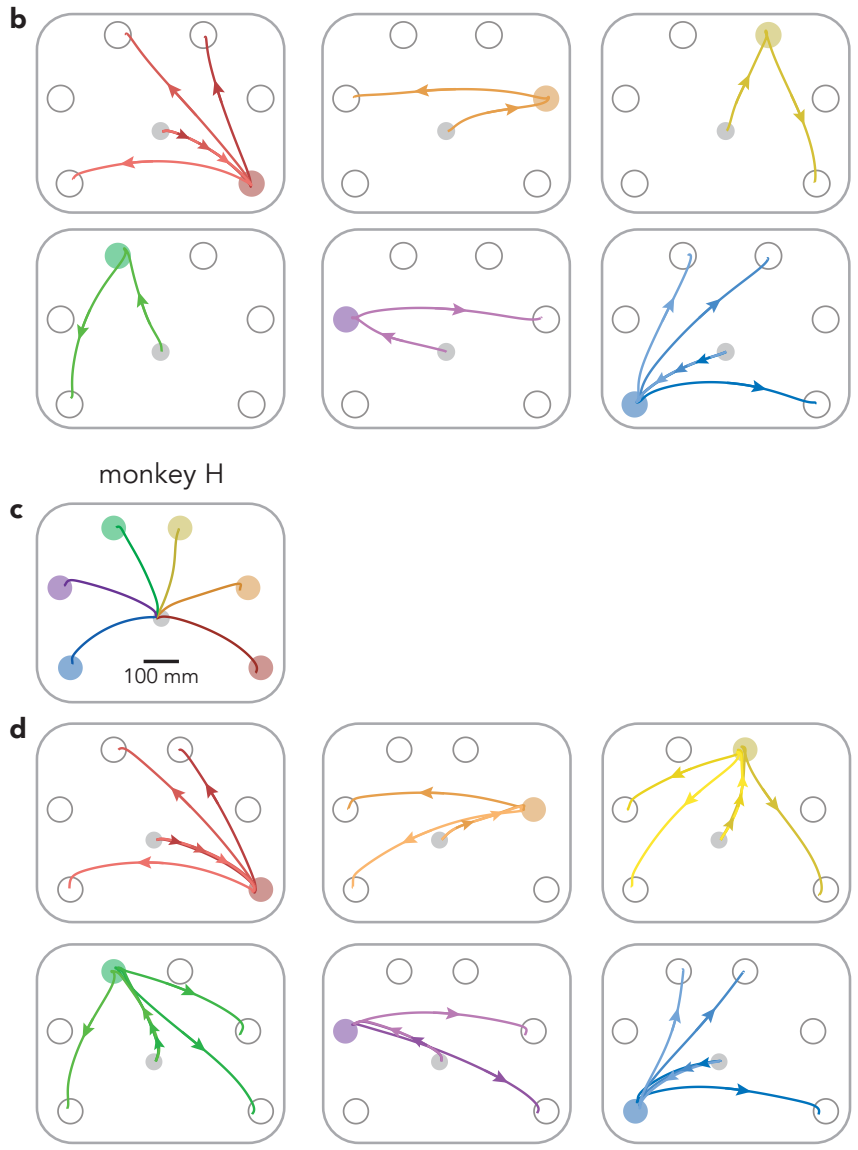

Extended Data Fig. 1 | Single and compound-reach conditions. a, Reach paths for single reach conditions (same as in Fig. 1a) for Monkey B. Paths are averaged across all trials and sessions. $\mathbf{b}$, Reach paths for all compound reach conditions performed by monkey B (a superset of those in Fig. 1a). Most first-target locations were used for only one compound reach condition, to maintain a reasonable trial-counts. This was necessary because monkey $B$ performed delayed double-reach conditions using three instructed pauses, which added to the total number of conditions performed. For similar reasons, delayed double-reach conditions employed a subset of the two-target combinations (those in red and blue) that were employed during compound reaches. c, Same as a, but for monkey H. For monkey H, the bottom-right and top-right targets were shifted slightly to the right and left (respectively) compared to the locations used for monkey B. This shift was necessary to prevent the animal's arm from blocking sight of the second target during certain conditions (the two monkeys were of different sizes and employed slightly different postures when reaching). $\mathbf{d}$, Same as $\mathbf{b}$, but for monkey $\mathrm{H}$. Monkey $\mathrm{H}$ performed a greater number of compound reach conditions than monkey B. 
bioRxiv preprint doi: https://doi.org/10.1101/2020.06.09.143040; this version posted June 10, 2020. The copyright holder for this preprint (which was not certified by peer review) is the author/funder, who has granted bioRxiv a license to display the preprint in perpetuity. It is made available under aCC-BY-NC-ND 4.0 International license.

\section{Extended Data Fig. 2}

a

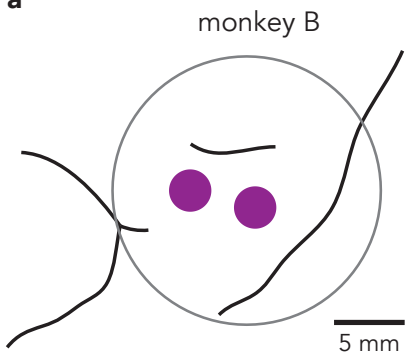

b

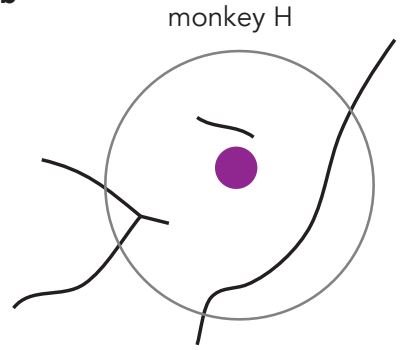

Extended Data Fig. 2 | Recording locations. a, Location of recording chamber (grey) and recording area (purple) relative to arcuate sulcus, pre-central dimple, and central sulcus (black, left to right). $\mathbf{b}$, Same as a but for Monkey $\mathrm{H}$. 
bioRxiv preprint doi: https://doi.org/10.1101/2020.06.09.143040; this version posted June 10,2020. The copyright holder for this preprint (which was not certified by peer review) is the author/funder, who has granted bioRxiv a license to display the preprint in perpetuity. It is made available under aCC-BY-NC-ND 4.0 International license.

\section{Extended Data Fig. 3}
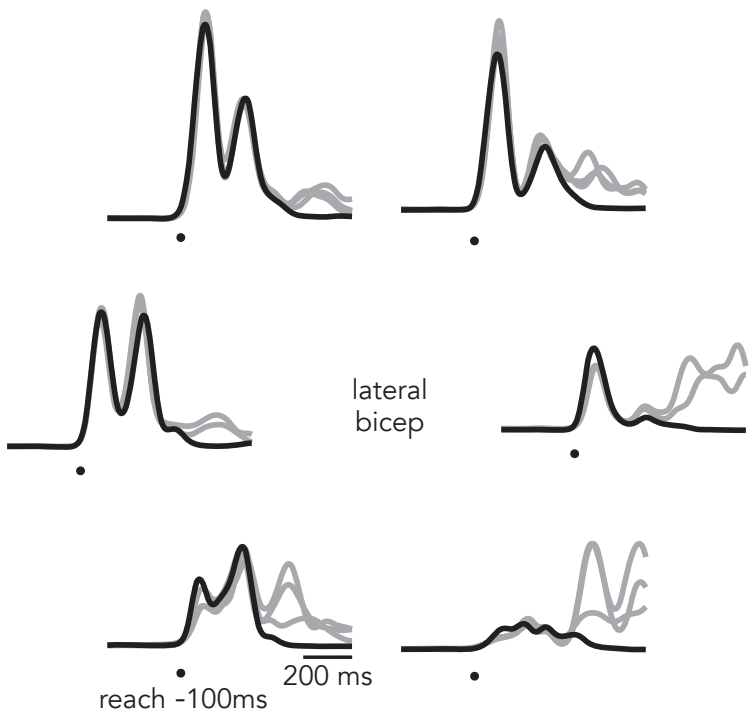

Extended Data Fig. 3 | Additional example EMG. Mean EMG for single (black) and compound (grey) reach conditions, Monkey $\mathrm{H}$. 


\section{Extended Data Fig. 4}

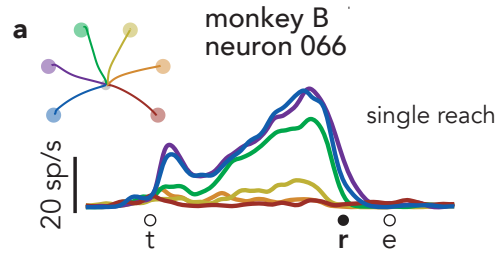

b

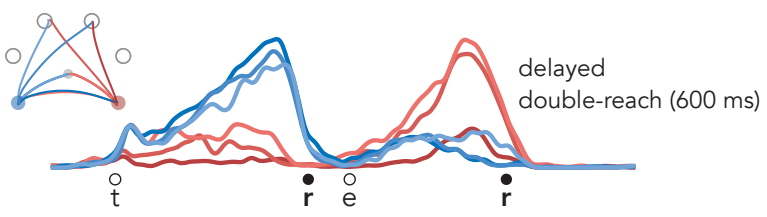

c

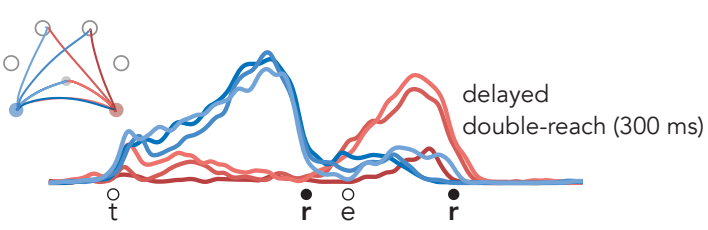

d

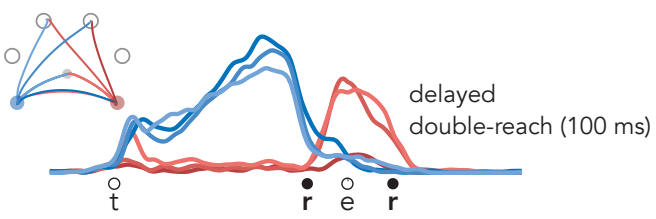

e

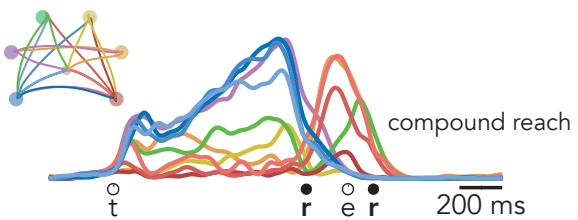

Extended Data Fig. 4 | Activity of neuron 066 (Monkey B) across all conditions. a, Response during single-reach conditions. This panel plots the same neural data as in Fig. 3a, but for all single reaches. $\mathbf{b}$, Response during delayed double-reaches with a $600 \mathrm{~ms}$ instructed pause. This panel plots the same neural data as in Fig. 3b. c, Response during delayed double-reaches with a $300 \mathrm{~ms}$ instructed pause. $\mathbf{d}$, Response during delayed double-reaches with a $100 \mathrm{~ms}$ instructed pause. e, Response during all compound reach conditions. The traces in this panel are a superset of those in Fig. 3c. 
bioRxiv preprint doi: https://doi.org/10.1101/2020.06.09.143040; this version posted June 10, 2020. The copyright holder for this preprint (which was not certified by peer review) is the author/funder, who has granted bioRxiv a license to display the preprint in perpetuity. It is made available under aCC-BY-NC-ND 4.0 International license.

\section{Extended Data Fig. 5}

a

monkey $\mathrm{H}$

neuron 322
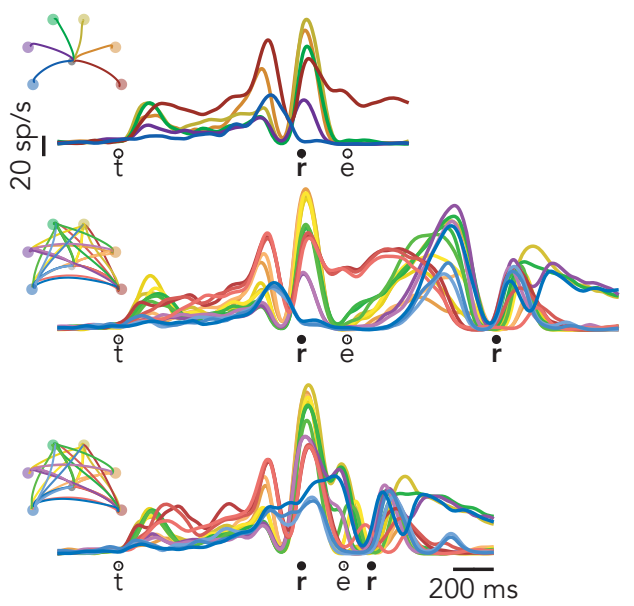

b
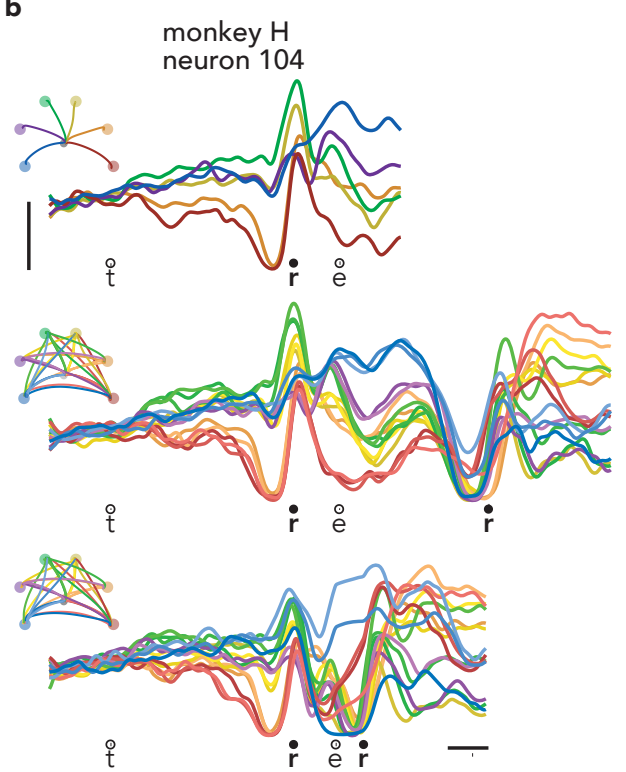

c
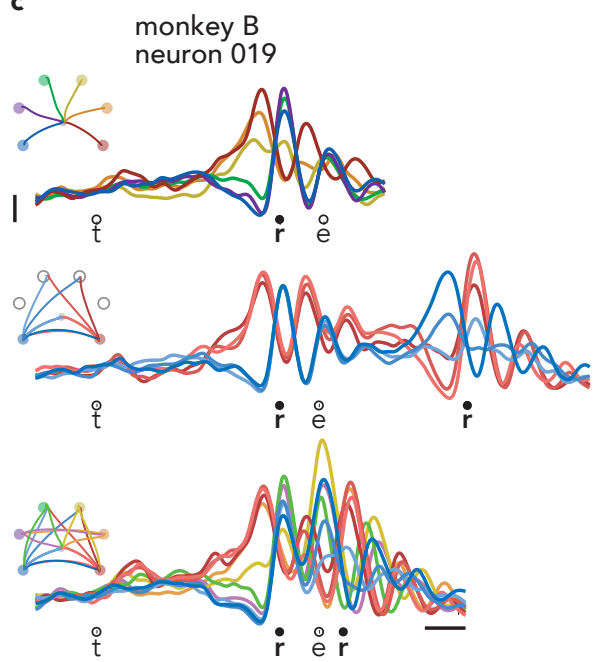

d
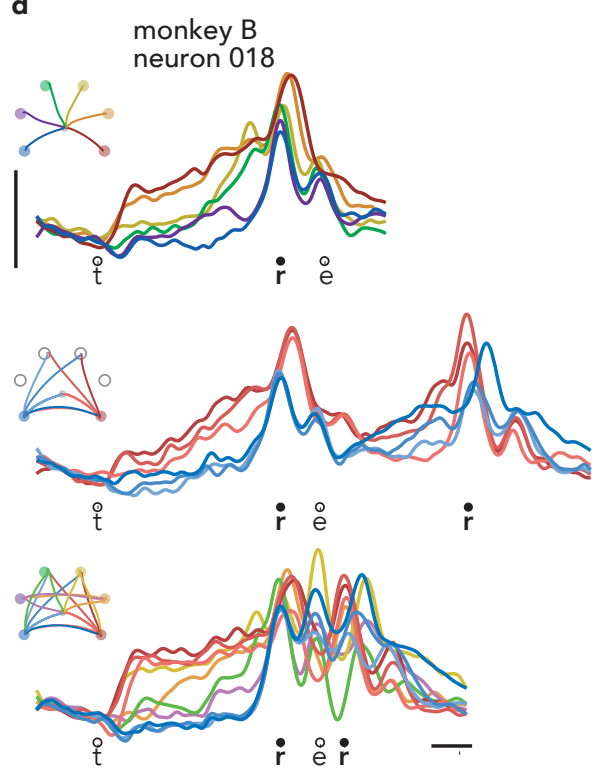

Extended Data Fig. 5 | Responses of four exemplar motor cortex neurons. a, Response of neuron 322, recorded from Monkey $\mathrm{H}$. These are the same neural data as in Fig. 3d-f, but are plotted here for all single reaches and all two-reach combinations. $\mathbf{b}-\mathbf{d}$, Responses of three additional example neurons. As is typical, these neurons are active during both the delay and execution-epochs of single reaches. It is thus difficult to determine via inspection whether there is a second bout of preparation during compound reaches. 
bioRxiv preprint doi: https://doi.org/10.1101/2020.06.09.143040; this version posted June 10, 2020. The copyright holder for this preprint (which was not certified by peer review) is the author/funder, who has granted bioRxiv a license to display the preprint in perpetuity. It is made available under aCC-BY-NC-ND 4.0 International license.

\section{Extended Data Fig. 6}

a

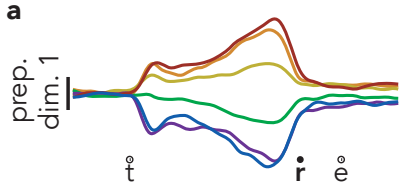

b
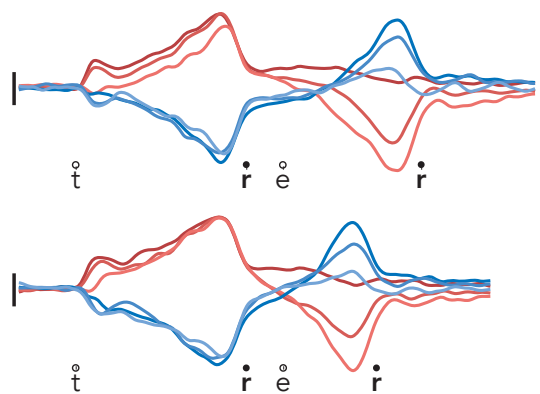

d

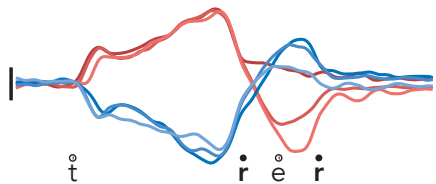

e

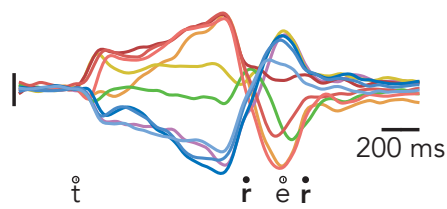

f

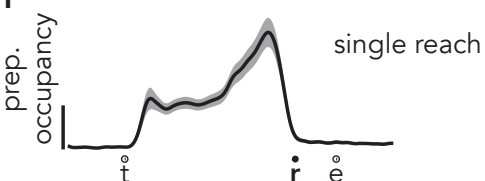

g

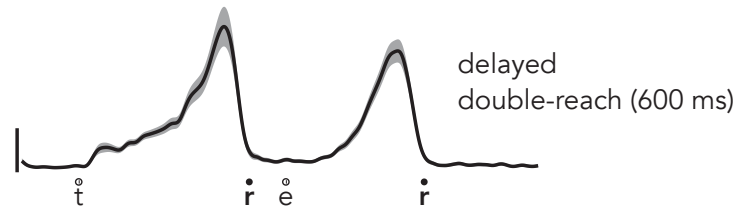

h

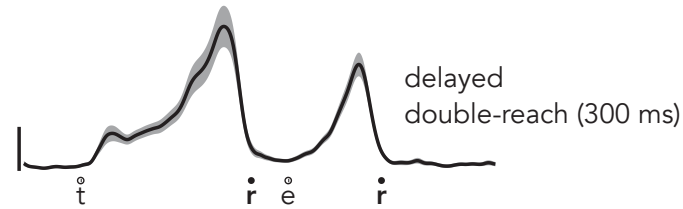

i

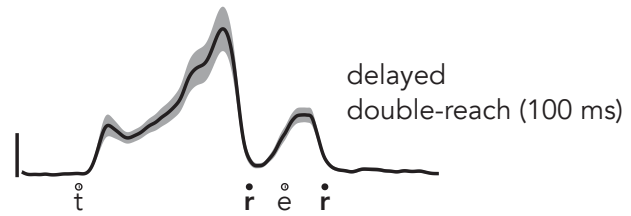

j

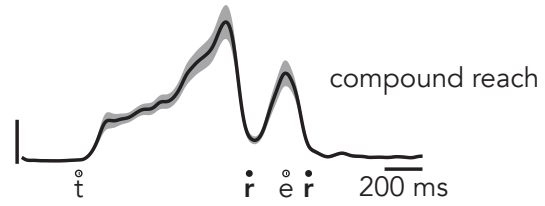

Extended Data Fig. 6 | Time-course of activity in preparatory dimensions during all conditions, monkey B. a, Projections of population activity during single reach conditions onto the first preparatory dimension. This panel plots the same data shown in Fig. 4a. b, Activity in the first preparatory dimension during delayed double-reach conditions with a $600 \mathrm{~ms}$ instructed pause. This panel plots the same data shown in Fig. 4b. c, Activity in the first preparatory dimension during delayed double-reach conditions with a 300 ms instructed pause. d, Activity in the first preparatory dimension during delayed double-reach conditions with a $100 \mathrm{~ms}$ instructed pause. e, Activity in the first preparatory dimension during compound reach conditions. This panel plots the same data shown in Fig. 4c. f, Occupancy of all 20 preparatory dimensions during single reach conditions. This panel plots the same data shown in Fig. 4d. g, Occupancy of preparatory dimensions during delayed double-reach conditions with a $600 \mathrm{~ms}$ instructed pause. This panel plots the same data shown in Fig. $4 \mathrm{e} . \mathbf{h}$, Occupancy of preparatory dimensions during delayed double-reach conditions with a $300 \mathrm{~ms}$ instructed pause. i, Occupancy of preparatory dimensions during delayed-double reaches with a $100 \mathrm{~ms}$ instructed pause. j, Occupancy of preparatory dimensions during compound reach conditions. This panel plots the same data shown in Fig. $4 f$. 


\section{Extended Data Fig. 7}

a

monkey B

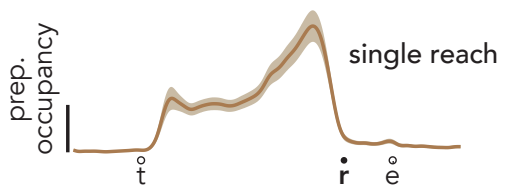

b

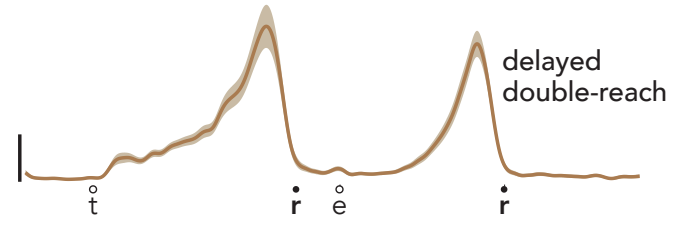

c

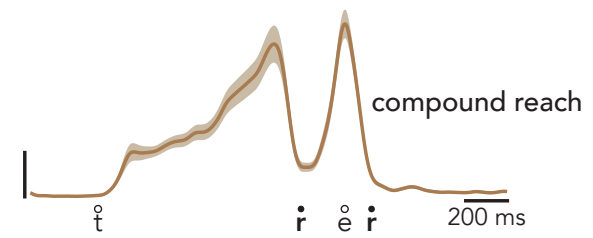

d

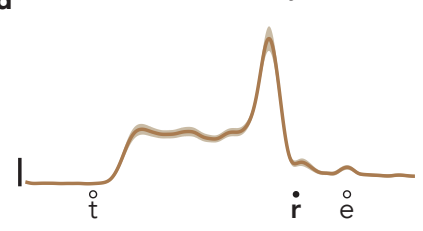

e

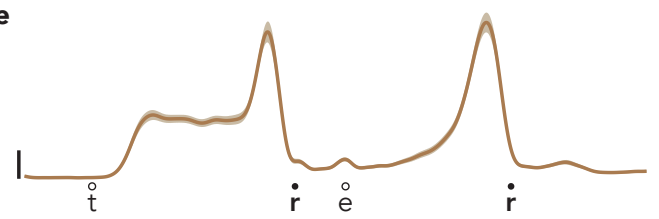

f

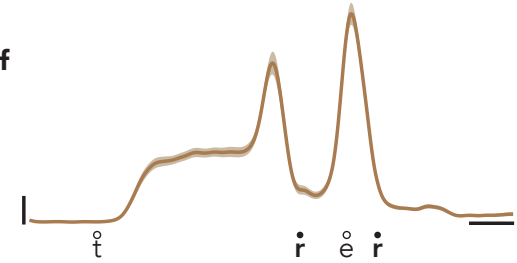

Extended Data Fig. 7 | Defining the preparatory dimensions using all preparatory epochs. a, Similar to Fig. $4 \mathrm{~d}$, this panel plots the occupancy of 20 preparatory dimensions during single reach conditions. However, these dimensions were found using dwell-period activity from compound reach conditions (activity within a $40 \mathrm{~ms}$ window beginning $140 \mathrm{~ms}$ before the onset of the second reach) in addition to activity used to define the preparatory dimensions in Fig. 4d-f. Data shown are from monkey B. b, Occupancy of the same preparatory dimensions during delayed double-reach conditions. c, Occupancy of the same preparatory dimensions during compound reach conditions. This panel plots the same data shown in Fig. 4g. d-f, Same as a-c, but data are from monkey $\mathrm{H}$. 


\section{Extended Data Fig. 8}
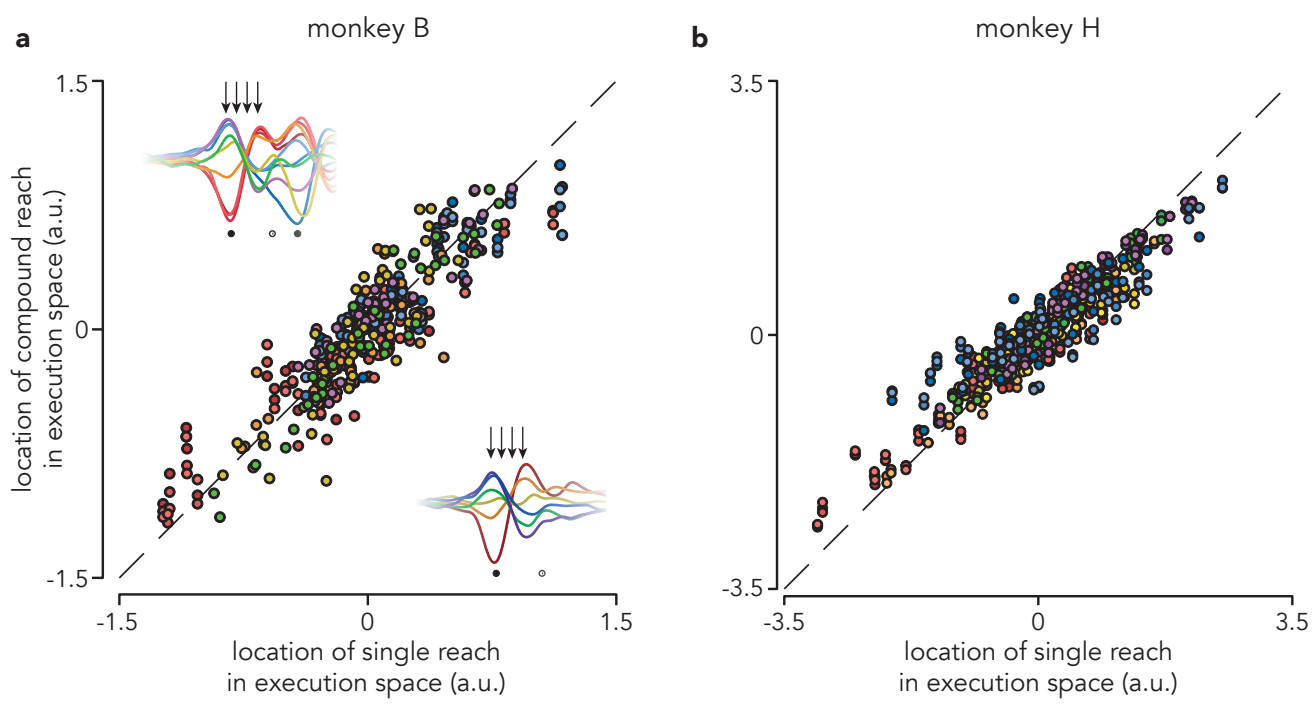

Extended Data Fig. 8 | Patterns of execution-related activity. a, Comparison of activity within the top 10 execution dimensions during single and compound reach conditions. Similar to Fig. $5 c$, each marker represents the activity (in 1 of 10 execution dimensions, at a single point in time) of a pair of conditions that share a first reach. Unlike Fig. 5, here, we plot activity from 4 time points (50 ms intervals, starting 25 ms before reach onset). Data shown are from monkey B. $\mathbf{b}$, same as $\mathbf{a}$, but data are from monkey $\mathrm{H}$. Although activity from the first reach of compound reaches was not used to define the preparatory and execution dimensions, these dimensions explained comparable amounts of variance during the first reach epoch of both single reaches and compound reaches. These dimensions explained $87 \%$ and $78 \%$ as much of the first reach epoch variance during compound reaches as was explained during single reaches, monkey $\mathrm{B}$ and monkey $\mathrm{H}$, respectively. 
bioRxiv preprint doi: https://doi.org/10.1101/2020.06.09.143040; this version posted June 10, 2020. The copyright holder for this preprint (which was not certified by peer review) is the author/funder, who has granted bioRxiv a license to display the preprint in perpetuity. It is made available under aCC-BY-NC-ND 4.0 International license.

\section{Extended Data Fig. 9}

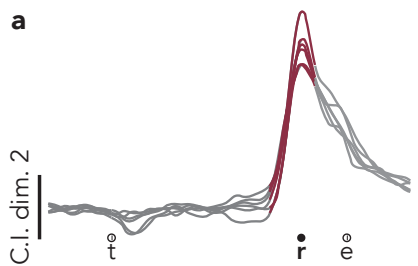

b
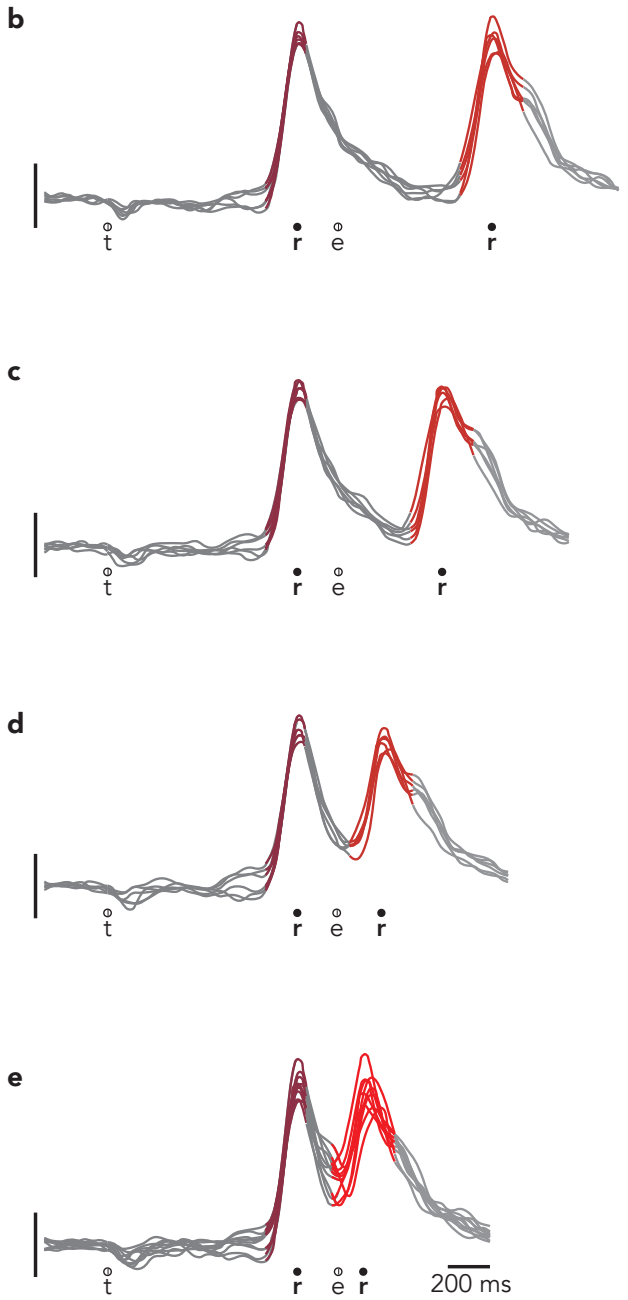

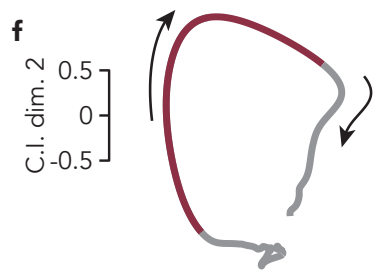

$\begin{array}{rll}-0.5 & 0 & 0.5\end{array}$

C.I. $\operatorname{dim} .1$

g

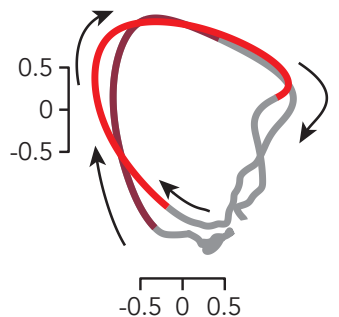

h

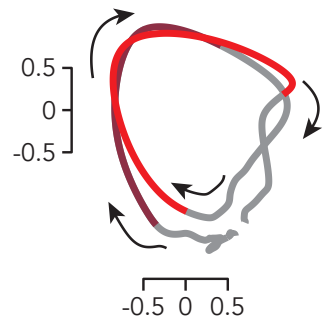

i
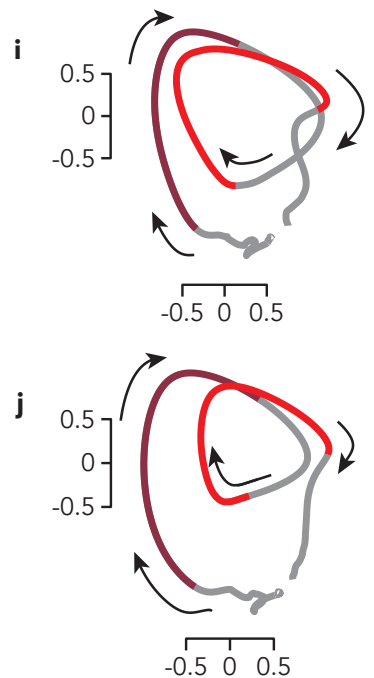

Extended Data Fig. 9 | Activity within condition-invariant dimensions during all conditions, monkey B. a, Projections of population activity from single reach conditions onto a single condition-invariant dimension. As in Fig. 6, traces are colored to highlight peri-reach activity. This panel plots the same data shown in Fig. 6a, top. b, Same as a but for delayed double-reaches with a $600 \mathrm{~ms}$ instructed pause. This panel plots the same data shown in Fig. 6b, top. c-d, Same as b but for delayed double-reaches with $300 \mathrm{~ms}$ (c) and $100 \mathrm{~ms}$ (d) instructed delays. e, Same as a but for compound reaches. This panel plots the same data shown in Fig. 6c, top. f, Average (across-condition) activity in two condition-invariant dimensions during single reach conditions. This panel plots the same data shown in Fig. 6a, bottom. g, Same as $\mathbf{f}$ but for delayed double-reaches with a $600 \mathrm{~ms}$ instructed pause. This panel plots the same data shown in figure 6b, bottom. h-i, Same as $\mathbf{g}$ but for delayed double-reaches with $300 \mathrm{~ms}$ (h) and $100 \mathrm{~ms}$ (i) instructed delays. j, Same as $\mathbf{f}$ but for compound reaches. This panel plots the same data shown in Fig. 6c, bottom. 